\title{
Existence of solution for a free boundary problem in a nonlinear piecewise homogeneous medium $\left({ }^{1}\right)$
}

by

\author{
A. BERMÚDEZ, M. C. MUÑIZ and P. QUINTELA \\ Department of Applied Mathematics, \\ University of Santiago de Compostela, 15706 Santiago, Spain
}

\begin{abstract}
A free boundary problem arising from the bidimensional thermal modelling of aluminium electrolytic cells is studied. The medium is assumed piecewise homogeneous and nonlinear. A fixed domain method is proposed which leads to a weak formulation of the problem. Existence of weak solution is proved by regularizing the contact condition between the homogeneous subdomains and passing to the limit.

(C) 1998 L'Association Publications de l'Institut Henri Poincaré. Published by Elsevier B.V. All rights reserved

Key words: Free boundary, aluminium electrolysis, piecewise homogeneous media, weak solution, existence.
\end{abstract}

RÉSumÉ. - Dans cet article on étudie un problème de frontière libre qui apparaît dans la modélisation thermoélectrique des cuves électrolytiques d'aluminium. Le domaine physique est supposé homogène par morceaux et nonlinéaire. On utilise une méthode de domaine fixe qui conduit à une formulation variationnelle du problème. L'existence de solution faible est démontré par régularisation de la condition de transmission entre les sousdomaines homogènes et passage à la limite.

(c) 1998 L'Association Publications de l'Institut Henri Poincaré. Published by Elsevier B.V. All rights reserved

(1) Partially supported by Xunta de Galicia (XUGA20701A93) and by INESPAL METAL S.A. (La Coruña, Spain) under contract. 


\section{INTRODUCTION}

In this paper, a free boundary problem motivated by the thermal modelling of an aluminium electrolytic cell is studied.

Aluminium is produced by reduction of alumina dissolved in an electrolytic bath based on molten cryolite (see [11]). This complex process, called Hall-Héroult, involves thermoelectrical and magnetohydrodynamical phenomena, electrochemical reactions, complex phase equilibria and so on (see [12]).

The Hall-Héroult process takes place in an electrolytic cell (see Fig. 1) which consists of a rectangular steel shell with an inner covering of insulating and refractory materials. Inside this, there is a linning of prebaked carbon cathode blocks with embedded steel current collector bars. Both the liquid metal and the electrolytic bath are upon these blocks. A frozen bath layer, the so-called ledge, protects the side wall of the cell from corrosive electrolyte. This ledge also reduces the heat loss from the cathode and works as a heat sink when extra power is supplied to the cell, thus playing a major role in the thermal behaviour of the cell.

The outline of this paper is as follows: in section 2 , we recall the main features characterizing the thermoelectrical behaviour of an electrolytic cell. The unknowns are the temperature, the electric potential and the profile of the ledge which becomes a free boundary.

Theoretical analysis of this problem is extremely difficult due to the coupling between thermal and electric equations, the nonhomogeneity of the domain, the physical nonlinearities and the free boundary. In [4], a discretized thermoelectrical problem is introduced and an iterative algorithm is used to compute the solution for a test example and real industrial electrolytic cells.

As a first step, in [6] we study the free boundary problem in the ledge which is both piecewise homogeneous and nonconductor, and consequently only the thermal phenomenon is considered. Both, existence and uniqueness of solution are demonstrated assuming that the ledge is linear (i.e. the thermal conductivity coefficients depend on space variables but not on temperature).

In the present paper we also study the thermal submodel. The difference with respect to the case considered in [6] is that now thermal conductivity also depends on temperature. This fact leads to a nonlinear diffusion term which makes more difficult the mathematical analysis. Indeed, since thermal conductivity also depends on the space variable, to avoid this nonlinearity by using a global Kirchhoff transformation is not allowed. To overcome this difficulty we use domain decomposition methods by 
considering two homogeneous subdomains (which can be distinguished in the ledge) corresponding to the levels of bath and aluminium.

In section 3 we introduce a weak formulation in a fixed domain. Mathematically, this problem is a stationary one phase Stefan problem with source at the free boundary (see [17]).

Section 4 is devoted to proving an existence theorem for an auxiliary problem, depending on a parameter, which regularizes the contact condition between the homogeneous subdomains. After setting some a priori estimates in section 5, existence of a weak solution for the thermal problem is proved in section 6 .

\section{THE THERMOELECTRICAL PROBLEM}

In this section we describe the thermoelectrical behaviour of the cathode of an aluminium electrolytic cell.

The voltage drop between the anode and the cathode causes an increasing of the temperature due to the Joule effect. Likewise, the potential distribution of the electrolytic cell depends on the temperature through the electrical conductivities of the materials. Therefore, from a mathematical point of view, the full problem couples both a thermal and an electrical problem, and it is similar to the so-called thermistor problem (see [13] and the references therein). However two additional difficulties appear in the present problem. Firstly, the domain of the model is not homogeneous and then physical parameters depend not only on temperature but on position $x$ as well. Secondly, there is a free boundary: the profile of the ledge, called $S$ in Fig. 1.

The boundary conditions for the electric problem are given by the knowledge of the current density through the cathodic bar. Moreover, the heat flux through the exterior boundaries due to the losses by convection an radiation leads to the boundary conditions for the thermal problem.

The ledge, being a nonconductor, is actually a fundamental part of the cell from the thermal point of view. In the recent years, several attempts have been made to determine the heat flux through the surface $S$ (see [1], [18]). Since the temperature is almost uniform in the liquid phase due to the strong horizontal flow caused by the electromagnetic field, we assume that the temperature, $T$, is equal to the solidus temperature of the bath, called $T_{s}$, in $S$. The heat flux on $S$ is given by

$$
k(x, T) \frac{\partial T}{\partial n}=h\left(x_{2}\right) n_{1}(x),
$$




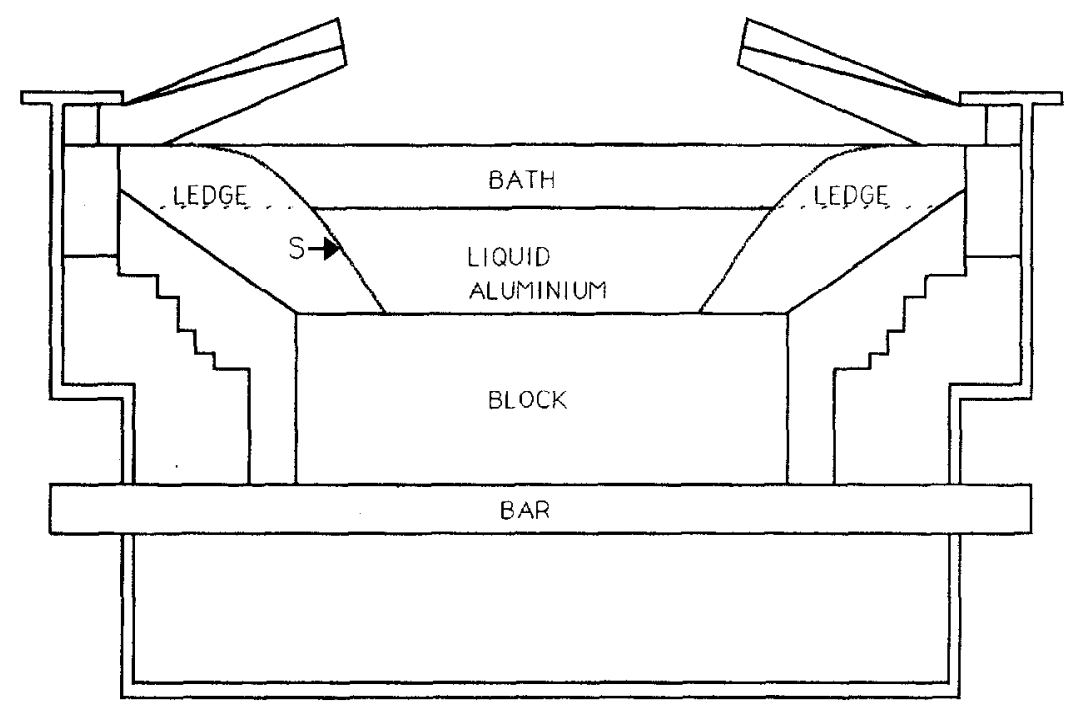

Fig. 1. - A section of the cell cathode.

where $k$ is the thermal conductivity depending both on the space variable $x=\left(x_{1}, x_{2}\right)$ and on the temperature, $n_{1}$ represents the first component of the outward unit normal vector to the ledge at $S$ at point $x$ and $h\left(x_{2}\right)$ is a function to be given which only depends on $x_{2}$. In practice, $h$ has to be identified from experimental measurements because it depends on factors as the electrolyte composition. In [3], a method to identify the function $h$ from experimental measurements of the ledge profile is developed. Including $n_{1}$ is not only convenient from the mathematical point of view but it also makes sense from the physical one because the heat transfer depends on the slope of the free boundary: the greater the slope the greater the heat transfer.

In [5], this full thermoelectrical problem is discretized using pentahedral finite elements of six degrees of freedom and numerical results are given for real industrial situations.

The difficulties appearing on the theoretical treatment of this coupled problem, as the nonlinearities on the physical characteristics of the materials or the free boundary, lead us to consider a simplified problem taking place on the (unknown!) domain occupied by the ledge.

\section{STATEMENT OF THE PROBLEM}

As a approach to the theoretical study of the full thermoelectrical problem, we consider a simplified bidimensional submodel. The ledge is the domain 
where the problem is now posed; it is formed by two layers corresponding to the bath and the metal lcvels and, as a conscquence, the thermal conductivity is different in these two levels (see Fig. 2). Since it is a nonconductor material, release of heat by Joule effect does not occur and then we can consider only the thermal part of the problem which becomes a one phase Stefan problem with source at the free boundary (see [17]). A similar problem is developed in [16] for the evolutionary and multiphase version but it does not cover the present situation.

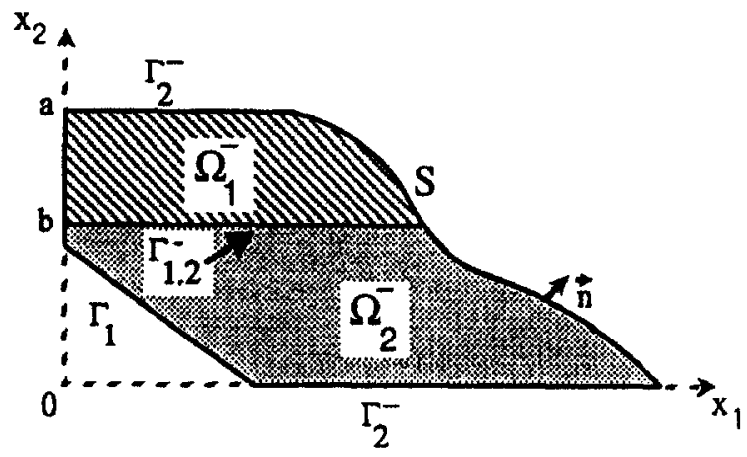

Fig. 2. $-\Omega^{-}$domain.

Let $\Omega^{-}$be the ledge. We assume that $\Omega^{-}$can be written as

$$
\Omega^{-}=\left\{\left(x_{1}, x_{2}\right) \in \mathbb{R}^{2}, 0<x_{2}<a, f_{1}\left(x_{2}\right)<x_{1}<f_{2}\left(x_{2}\right)\right\},
$$

where $a$ is a positive real number, $f_{i}, i=1,2$ are Lipschitz functions and

$$
f_{1}\left(x_{2}\right)<f_{2}\left(x_{2}\right), \forall x_{2} \in[0, a] .
$$

Actually, $f_{2}$ is an unknown function corresponding to the free boundary.

The solidified bath and metal are denoted by $\Omega_{1}^{-}$and $\Omega_{2}^{-}$, respectively and we suppose that they are given by

$$
\begin{aligned}
& \Omega_{1}^{-}=\Omega^{-} \cap\left\{\left(x_{1}, x_{2}\right) \in \mathbb{R}^{2}, b<x_{2}<a\right\}, \\
& \Omega_{2}^{-}=\Omega^{-} \cap\left\{\left(x_{1}, x_{2}\right) \in \mathbb{R}^{2}, 0<x_{2}<b\right\},
\end{aligned}
$$

where $b$ is a positive real number such that $b<a$ (see Fig. 2).

Let $\Gamma_{1,2}^{-}=\partial \Omega_{1}^{-} \cap \partial \Omega_{2}^{-}$and $\Gamma_{1}$ and $S$ be the graphs of the functions $f_{1}$ and $f_{2}$, respectively. Finally,

$$
\Gamma_{2}^{-}=\partial \Omega^{-} \cap\left(\left[x_{2}=0\right] \cup\left[x_{2}=a\right]\right) .
$$

Vol. $15, \mathrm{n}^{\circ} 4-1998$. 
Notice that $\Gamma_{1} \cup \Gamma_{2}^{-}$is the part of the boundary of $\Omega^{-}$different from the free boundary $S$. We denote

$$
S_{i}=S \cap \partial \Omega_{i}^{-}, i=1,2
$$

Since $\Omega^{-}$is piecewise homogeneous, the thermal conductivity can be written as follows:

$$
k(x, T)= \begin{cases}k_{1}(T(x)) & \text { if } x \in \Omega_{1}^{-} \\ k_{2}(T(x)) & \text { if } x \in \Omega_{2}^{-}\end{cases}
$$

On the other hand, a Robin boundary condition is assumed on $\Gamma_{1}$ involving a convective coefficient $\alpha$ and the convective temperature of surroundings, $T_{c}$.

We shall assume all along the following assumptions on the data (Hl) For $i=1,2, k_{i}(s): \mathbb{R} \rightarrow \mathbb{R}$ are continuous and there exist positive constants $k_{\text {min }}$ and $k_{\text {max }}$, such that $k_{\text {min }} \leq k_{i}(s) \leq k_{\text {max }}$.

(H2) $T_{s}$ is a positive constant.

(H3) $h \in L^{\infty}(0, a)$ is nonnegative where $a$ is the height of the domain (see Fig. 2).

(H4) The function $\alpha$ only depends on the space variable and belongs to $L^{\infty}\left(\Gamma_{1}\right)$. Moreover, $\alpha(x) \geq \alpha_{\min }>0$ a.e. on $\Gamma_{1}$.

(H5) $T_{c} \in L^{\infty}(0, a)$, with $0<T_{m i n} \leq T_{c}<T_{s}$ a.e. on $(0, a)$.

(H6) $h n_{1}+\alpha\left(T_{s}-T_{c}\right) \geq 0$ a.e. on $\Gamma_{1}$, where $n_{1}$ denotes the first component of the outward unit normal vector to $\Gamma_{1}$. We assume that $-1 \leq n_{1}<0$.

Physically, the assumption (H6) establishes an upper bound in the heat source at the free boundary. From the theoretical point of view, it is needed in order to prove that the solution of our problem is less or equal than $T_{s}$. In [5] and for a onedimensional version, solutions without this property are obtained if (H6) does not hold.

Throughout this paper we use standard notations for Sobolev spaces and norms. We also denote

$$
\begin{gathered}
H(\operatorname{div}, \Omega)=\left\{\mathbf{v} \in\left(L^{2}(\Omega)\right)^{2} ; \text { div } \mathbf{v} \in L^{2}(\Omega)\right\}, \\
H_{00}^{1 / 2}\left(\Gamma_{0}\right)=\left\{\mu \in L^{2}\left(\Gamma_{0}\right) ; \exists z \in H^{1}(\Omega)\right. \\
\left.\quad \text { such that }\left.z\right|_{\Gamma_{0}}=\mu \text { and }\left.z\right|_{\left(\partial \Omega \backslash \Gamma_{0}\right)}=0\right\},
\end{gathered}
$$

where $\Gamma_{0}$ is an open set of the boundary of $\Omega$. 
Let us consider the following free boundary problem:

- Problem (P)

Find $T_{i}$ in $H^{1}\left(\Omega_{i}^{-}\right)$and $S_{i}, i=1,2$ such that

$$
\begin{aligned}
-\nabla \cdot\left(k_{i}\left(T_{i}(x)\right) \nabla T_{i}(x)\right) & =0 \text { in } \Omega_{i}^{-}, \\
k_{i}\left(T_{i}(x)\right) \frac{\partial T_{i}}{\partial n}(x) & =0 \text { on } \Gamma_{2}^{-} \cap \partial \Omega_{i}^{-}, \\
T_{i}(x) & =T_{s} \text { on } S_{i}, \\
k_{i}\left(T_{i}(x)\right) \frac{\partial T_{i}}{\partial n}(x) & =h\left(x_{2}\right) n_{1}(x) \text { on } S_{i}, \\
k_{i}\left(T_{i}(x)\right) \frac{\partial T_{i}}{\partial n}(x)+\alpha\left(x_{2}\right)\left(T_{i}(x)-T_{c}\left(x_{2}\right)\right) & =0 \text { on } \Gamma_{1} \cap \partial \Omega_{i}^{-},
\end{aligned}
$$

for $i=1,2$. Moreover, we must impose the transmission conditions on $\Gamma_{1,2}^{-}$:

$$
\begin{gathered}
T_{1}(x)=T_{2}(x), \\
k_{1}\left(T_{1}(x)\right) \frac{\partial T_{1}}{\partial n_{\Omega_{\perp}^{-}}}(x)+k_{2}\left(T_{2}(x)\right) \frac{\partial T_{2}}{\partial n_{\Omega_{2}^{-}}}(x)=0,
\end{gathered}
$$

$n_{\Omega^{-}}$being the outward unit normal vector to $\Omega_{i}^{-}, i=1,2$. The conditions (3.15) and (3.16) express the requirement for the temperature and the heat flux not to have jumps on $\Gamma_{1,2}^{-}$.

Equality (3.10) holds in the distributional sense and then $k_{i}\left(T_{i}(x)\right) \nabla T_{i}(x)$ belongs to $H\left(d i v, \Omega_{i}^{-}\right), i=1,2$. The boundary condition (3.11) holds on $\left(H_{00}^{1 / 2}\left(\Gamma_{2}^{-} \cap \partial \Omega_{i}^{-}\right)\right)^{\prime}$, and analogously with (3.13), (3.14) and (3.16).

For theoretical and numerical purposes, it is interesting to embed the problem ( $\mathrm{P}$ ) into another one defined in a fixed domain. For this purpose, we consider the sets $\Omega_{1}^{0}$ and $\Omega_{2}^{0}$ called the fictitious domains (see Fig. 3).

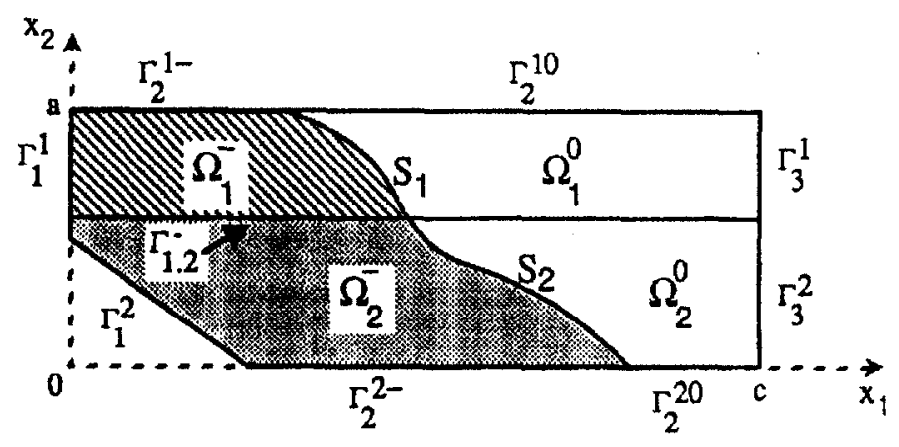

Fig. 3. $-\Omega$ domain. 
We define $\Omega_{i}$ as the interior of the set $\Omega_{i}^{-} \cup S_{i} \cup \Omega_{i}^{0}$, and

$$
\begin{aligned}
\Gamma_{1,2} & =\partial \Omega_{1} \cap \partial \Omega_{2}, \\
\Gamma_{1}^{i} & =\Gamma_{1} \cap \partial \Omega_{i}, \\
\Gamma_{2}^{i-} & =\Gamma_{2}^{-} \cap \partial \Omega_{i}^{-}, \\
\Gamma_{3}^{i} & =\left\{\left(x_{1}, x_{2}\right) \in \partial \Omega_{i} ; x_{1}=c\right\}, \Gamma_{3}=\Gamma_{3}^{1} \cup \Gamma_{3}^{2} \\
\Gamma_{2}^{i 0} & =\partial \Omega_{i} \backslash\left(\Gamma_{1}^{i} \cup \Gamma_{1,2} \cup \Gamma_{3}^{i} \cup \Gamma_{2}^{i-}\right), \\
\Gamma_{2}^{i} & =\Gamma_{2}^{i-} \cup \Gamma_{2}^{i 0}, \Gamma_{2}=\Gamma_{2}^{1} \cup \Gamma_{2}^{2},
\end{aligned}
$$

where the meaning of $c$ is clear from Fig. 3. We define $\Omega$ as the interior of the set $\Omega_{1} \cup \Omega_{2} \cup \Gamma_{1,2}$ with boundary $\Gamma=\Gamma_{1} \cup \Gamma_{2} \cup \Gamma_{3}$. Moreover we set

$$
\mathcal{H}^{1}(\Omega)=H^{1}\left(\Omega_{1}\right) \times H^{1}\left(\Omega_{2}\right),
$$

with the standard product norm, i.e.

$$
\left\|\left(u_{1}, u_{2}\right)\right\|=\left(\left\|u_{1}\right\|_{1,2, \Omega_{1}}^{2}+\left\|u_{2}\right\|_{1,2, \Omega_{2}}^{2}\right)^{1 / 2}
$$

As $H^{1}(\Omega)$ is continuously imbedded into $\mathcal{H}^{1}(\Omega)$, hereafter a function $T \in H^{1}(\Omega)$ will be denoted by $\left(T_{1}, T_{2}\right) \in \mathcal{H}^{1}(\Omega)$ with $T_{i}=\left.T\right|_{\Omega_{i}}, i=1,2$.

We consider the weak problem:

\section{- Problem (WP)}

Find $T=\left(T_{1}, T_{2}\right) \in H^{1}(\Omega), q_{i} \in L^{\infty}\left(\Omega_{i}\right), i=1,2$ such that

$$
\begin{gathered}
\sum_{i=1}^{2} \int_{\Omega_{i}} k_{i}\left(T_{i}\right) \nabla T_{i} . \nabla z_{i} d x+\sum_{i=1}^{2} \int_{\Omega_{i}} h q_{i} \frac{\partial z_{i}}{\partial x_{1}} d x+\sum_{i=1}^{2} \int_{\Gamma_{1}^{i}} \alpha\left(T_{i}-T_{c}\right) z_{i} d \Gamma \\
=\sum_{i=1}^{2} \int_{\Gamma_{3}^{i}} h z_{i} d \Gamma, \quad \forall\left(z_{1}, z_{2}\right) \in H^{1}(\Omega) \\
q_{i} \in H\left(T_{i}-T_{s}\right), \quad i=1,2,
\end{gathered}
$$

where $H$ denotes the multivalued Heaviside function given by

$$
H(r)=\left\{\begin{array}{ccc}
0 & \text { if } & r<0 \\
{[0,1]} & \text { if } & r=0 \\
1 & \text { if } & r>0
\end{array}\right.
$$


For a solution of the problem (WP), let the sets $\Omega^{-}, S, \Omega_{i}^{-}, \Omega_{i}^{0}, \Gamma_{2}^{-}$ and $\Gamma_{1.2}^{-}$be defined by

$$
\begin{aligned}
\Omega^{-} & =\left\{x \in \Omega: T(x)<T_{s}\right\} \\
S & =\partial \Omega^{-} \cap \Omega \\
\Omega_{i}^{-} & =\Omega^{-} \cap \Omega_{i} \\
\Omega_{i}^{0} & =\Omega_{i} \backslash\left(\Omega_{i}^{-} \cup S_{i}\right) \\
\Gamma_{2}^{-} & =\Gamma_{2} \cap \partial \Omega^{-} \\
\Gamma_{1,2}^{-} & =\partial \Omega_{1}^{-} \cap \partial \Omega_{2}^{-}
\end{aligned}
$$

$S_{i}, \Gamma_{2}^{i-}$ and $\Gamma_{2}^{i 0}$ being defined in (3.6), (3.19) and (3.21) respectively.

Notice that $q_{i}$ is equal to zero in $\Omega_{i}^{-}$by (3.26).

Remark 3.1. - If the solution $\left(T_{1}, T_{2}\right)$ of the problem (WP) is continuous and less or equal than $T_{s}$ and $S$ has twodimensional Lebesgue measure zero, then the set equality

$$
\Gamma_{1,2}=\Gamma_{1,2}^{0} \cup \Gamma_{1,2}^{-}
$$

holds where $\Gamma_{1,2}^{0}=\partial \Omega_{1}^{0} \cap \partial \Omega_{2}^{0}$ (see [15] for further details).

Proposition 3.1. - If there exists a regular solution $\left(T_{1}, T_{2}, q_{1}, q_{2}\right)$ of the problem (WP) such that $T_{i}(x) \leq T_{s}$ a.e. in $\Omega_{i}$ and furthermore $\Gamma_{1}^{i} \subset \partial \Omega_{i}^{-}$, $\Gamma_{3}^{i} \subset \partial \Omega_{i}^{0}$ and $\Omega_{i}^{-}$and $\Omega_{i}^{0}$ are open sets with Lipschitz boundary, $(i=1,2)$, then $\left(T_{1}, T_{2}, q_{1}, q_{2}, S\right)$ is a solution of the problem:

$$
\begin{aligned}
-\nabla .\left(k_{i}\left(T_{i}(x)\right) \nabla T_{i}(x)\right) & =0 \text { in } \Omega_{i}^{-}, \\
T_{i}(x)<T_{s}, \quad q_{i}(x) & =0 \text { in } \Omega_{i}^{-}, \\
-h\left(x_{2}\right) \frac{\partial q_{i}}{\partial x_{1}}(x) & =0 \text { in } \Omega_{i}^{0}, \\
T_{i}(x)=T_{s}, \quad 0 \leq q_{i}(x) & \leq 1 \text { in } \Omega_{i}^{0}, \\
T_{i}(x) & =T_{s} \text { on } S_{i}, \\
k_{i}\left(T_{i}(x)\right) \frac{\partial T_{i}}{\partial n}(x) & =h\left(x_{2}\right) q_{i}(x) n_{1}(x) \text { on } S_{i}, \\
k_{i}\left(T_{i}(x)\right) \frac{\partial T_{i}}{\partial n}(x) & =0 \text { on } \Gamma_{2}^{i}, \\
h\left(x_{2}\right) q_{i}(x) & =h\left(x_{2}\right) \text { on } \Gamma_{3}^{i}, \\
k_{i}\left(T_{i}(x)\right) \frac{\partial T_{i}}{\partial n}(x) & +\alpha\left(x_{2}\right)\left(T_{i}(x)-T_{c}\left(x_{2}\right)\right)=0 \text { on } \Gamma_{1}^{i}, \\
T_{1}(x) & =T_{2}(x) \text { on } \Gamma_{1,2}, \\
k_{1}\left(T_{1}(x)\right) \frac{\partial T_{1}}{\partial n_{\Omega_{1}}}(x) & +k_{2}\left(T_{2}(x)\right) \frac{\partial T_{2}}{\partial n_{\Omega_{2}}}(x)=0 \text { on } \Gamma_{1,2},
\end{aligned}
$$


for $i=1,2$ and $n_{1}$ as in equation (2.1).

In (3.40) $q_{i}$ represents the trace of $q_{i}$ restricted to $\Omega_{i}^{0}$ on $S_{i}$.

Proof. - If we choose $z \in \mathcal{D}\left(\Omega_{i}^{-}\right)$(the usual space of functions of class $\mathcal{C}^{\infty}$ with compact support in $\left.\Omega_{i}^{-}\right)$and $z \subset \mathcal{D}\left(\Omega_{i}^{0}\right)$, we classically have (3.35) and (3.37) in $\mathcal{D}^{\prime}\left(\Omega_{i}^{-}\right)$and in $\mathcal{D}^{\prime}\left(\Omega_{i}^{0}\right)$, respectively.

By definition of $\Omega_{i}^{0}$ and $\Omega_{i}^{-}$, we have $T_{i}=T_{s}$ in $\Omega_{i}^{0}$ and $q_{i}=0$ in $\Omega_{i}^{-}$, respectively. Thus, by applying the Green formula, equation (3.25) implies

$$
\begin{aligned}
& \sum_{i=1}^{2} \int_{\Gamma_{1}^{i} \cup \Gamma_{2}^{i} \cup S_{i}} k_{i}\left(T_{i}\right) \frac{\partial T_{i}}{\partial n} z_{i} d \Gamma+\int_{\Gamma_{1,2}} k_{1}\left(T_{1}\right) \frac{\partial T_{1}}{\partial n_{\Omega_{1}}} z_{1} d \Gamma^{2} \\
& +\int_{\Gamma_{1,2}} k_{2}\left(T_{2}\right) \frac{\partial I_{2}}{\partial n_{\Omega_{2}}} z_{2} d \Gamma+\sum_{i=1}^{2} \int_{S_{i}} h q_{i}\left(-n_{1}\right) z_{i} d \Gamma+\sum_{i=1}^{2} \int_{\Gamma_{3}^{i}} h q_{i} z_{i} d \Gamma \\
& +\sum_{i=1}^{2} \int_{\Gamma_{1}^{i}} \alpha\left(T_{i}-T_{c}\right) z_{i} d \Gamma=\sum_{i=1}^{2} \int_{\Gamma_{i}^{i}} h z_{i} d \Gamma, \quad \forall\left(z_{1}, z_{2}\right) \in H^{1}(\Omega),
\end{aligned}
$$

taking into account that $\nabla T_{i}=0$ in $\Omega \backslash \Omega^{-}$. From (3.46), we deduce $(3.40),(3.41),(3.42),(3.43)$ and (3.45). Finally, since $\left(T_{1}, T_{2}\right) \in H^{1}(\Omega)$, (3.44) holds true.

Notice that if $\Omega_{i}^{0}$ is connected and $\Gamma_{3}^{i} \subset \partial \Omega_{i}^{0}$, (3.37) and (3.42) imply $h q_{i}=h$ in $\Omega_{i}^{0}$, and therefore from (3.40) we deduce (3.13).

The problem (WP) is similar to those arising in the dam problem (see [9]), and in the lubrication with cavitation problem (see [2], [10]). The differences lie in both the coefficients of the partial differential operators and the boundary conditions. In [6], an easier problem is considered, in that thermal conductivity does not depend on temperature and hence the differential operator in equation (3.10) is linear. For this problem, existence and uniqueness of solution are proved. However, the technique developed in that paper can not be directly applied to the present problem.

In the following sections we are concerned with existence of solution of the problem (WP). The proof is laborious and is based on defining a regularized problem using maximal monotone operators techniques.

\section{THE REGULARIZED PROBLEM}

In this section we introduce the regularized problem, called problem $\left(A P_{\lambda}\right)$. An existence result for this problem is given after both a Kirchhoff transformation and an approximation technique are used. 
Let us consider the operator

$$
\mathcal{A}: \mathcal{H}^{1}(\Omega) \times L^{\infty}\left(\Omega_{1}\right) \times L^{\infty}\left(\Omega_{2}\right) \rightarrow\left(\mathcal{H}^{1}(\Omega)\right)^{\prime}
$$

defined by

$$
\begin{gathered}
<\mathcal{A}\left(T_{1}, T_{2}, q_{1}, q_{2}\right),\left(z_{1}, z_{2}\right)>=\sum_{i=1}^{2} \int_{\Omega_{i}} k_{i}\left(T_{i}\right) \nabla T_{i} \cdot \nabla z_{i} d x \\
+\sum_{i=1}^{2} \int_{\Omega_{i}} h q_{i} \frac{\partial z_{i}}{\partial x_{1}} d x+\sum_{i=1}^{2} \int_{\Gamma_{1}^{i}} \alpha T_{i} z_{i} d \Gamma
\end{gathered}
$$

and the element $\mathcal{F}$ of $\left(\mathcal{H}^{1}(\Omega)\right)^{\prime}$ given by

$$
\mathcal{F}\left(z_{1}, z_{2}\right)=\sum_{i=1}^{2} \int_{\Gamma_{1}^{\prime}} \alpha T_{c} z_{i} d \Gamma+\sum_{i=1}^{2} \int_{\Gamma_{3}^{i}} h z_{i} d \Gamma
$$

We are able to prove the following

Proposition 4.2. - Let $\left(T_{1}, T_{2}, q_{1}, q_{2}\right)$ be an element of $\mathcal{H}^{1}(\Omega) \times L^{\infty}\left(\Omega_{1}\right) \times$ $L^{\infty}\left(\Omega_{2}\right)$ such that

$$
\begin{aligned}
\mathcal{F}-\mathcal{A}\left(T_{1}, T_{2}, q_{1}, q_{2}\right) & \in \gamma_{1,2}^{\star} \partial I_{\{0\}}\left(\gamma_{1,2}\left(T_{1}, T_{2}\right)\right) \\
q_{i} & \in H\left(T_{i}-T_{s}\right), i=1,2
\end{aligned}
$$

then $\left(T_{1}, T_{2}, q_{1}, q_{2}\right)$ is a solution of problem (WP), where:

- $\partial I_{\{0\}}$ is the subdifferential of $I_{\{0\}}$ which is the indicator function of the set $\{0\}$ in $H^{1 / 2}\left(\Gamma_{1,2}\right)$.

- $\gamma_{1,2}$ is defined by

$$
\begin{aligned}
\gamma_{1,2}: \mathcal{H}^{1}(\Omega) & \longrightarrow H^{1 / 2}\left(\Gamma_{1,2}\right) \\
\left(z_{1}, z_{2}\right) & \left.\rightarrow z_{1}\right|_{\Gamma_{1,2}}-z_{2} \mid \Gamma_{1,2}
\end{aligned}
$$

- $\gamma_{1,2}^{\star}$ is the adjoint operator of $\gamma_{1,2}$.

Proof. - Since $I_{H^{1}(\Omega)}=I_{\{0\}} \circ \gamma_{1,2}$, by using the chain rule of subdifferential calculus we obtain

$$
\mathcal{F}-\mathcal{A}\left(T_{1}, T_{2}, q_{1}, q_{2}\right) \in \partial I_{H^{1}(\Omega)}\left(T_{1}, T_{2}\right)
$$

Vol. 15, n ${ }^{\circ}$ 4-1998. 
Therefore,

$$
\begin{gathered}
<\mathcal{A}\left(T_{1}, T_{2}, q_{1}, q_{2}\right),\left(z_{1}, z_{2}\right)-\left(T_{1}, T_{2}\right)>+I_{H^{1}(\Omega)}\left(z_{1}, z_{2}\right)-I_{H^{1}(\Omega)}\left(T_{1}, T_{2}\right) \\
\geq \mathcal{F}\left(\left(z_{1}, z_{2}\right)-\left(T_{1}, T_{2}\right)\right), \quad \forall\left(z_{1}, z_{2}\right) \in \mathcal{H}^{1}(\Omega),
\end{gathered}
$$

and we deduce that $\left(T_{1}, T_{2}\right)$ belongs to $H^{1}(\Omega)$. Taking into account that $H^{1}(\Omega)$ is a subspace of $\mathcal{H}^{1}(\Omega)$, we easily deduce

$$
<\mathcal{A}\left(T_{1}, T_{2}, q_{1}, q_{2}\right),\left(z_{1}, z_{2}\right)>=\mathcal{F}\left(\left(z_{1}, z_{2}\right)\right), \forall\left(z_{1}, z_{2}\right) \in H^{1}(\Omega)
$$

and the proof is complete.

Taking into account (4.4), we define an auxiliary problem by replacing the maximal monotone operator $\partial I_{\{0\}}$ by its Yosida approximation given by

$$
\left(\partial I_{\{0\}}\right)_{\lambda}(s)=\frac{s}{\lambda} .
$$

\section{- Problem $\left(\mathbf{A P}_{\lambda}\right)$}

For a fixed $\lambda>0$, find $\left(T_{1}^{\lambda}, T_{2}^{\lambda}, q_{1}^{\lambda}, q_{2}^{\lambda}\right)$ in $\mathcal{H}^{1}(\Omega) \times L^{\infty}\left(\Omega_{1}\right) \times L^{\infty}\left(\Omega_{2}\right)$ such that

$$
\begin{aligned}
& \sum_{i=1}^{2} \int_{\Omega_{i}} k_{i}\left(T_{i}^{\lambda}\right) \nabla T_{i}^{\lambda} \cdot \nabla z_{i} d x \\
& +\sum_{i=1}^{2} \int_{\Omega_{i}} h q_{i}^{\lambda} \frac{\partial z_{i}}{\partial x_{1}} d x+\sum_{i=1}^{2} \int_{\Gamma_{1}^{i}} \alpha\left(T_{i}^{\lambda}-T_{c}\right) z_{i} d \Gamma \\
& +\int_{\Gamma_{1,2}} \frac{\left(T_{1}^{\lambda}-T_{2}^{\lambda}\right)}{\lambda}\left(z_{1}-z_{2}\right) d \Gamma \\
& =\sum_{i=1}^{2} \int_{\Gamma_{3}^{i}} h z_{i} d \Gamma, \forall\left(z_{1}, z_{2}\right) \in \mathcal{H}^{1}(\Omega), \\
& q_{i}^{\lambda} \in H\left(T_{i}^{\lambda}-T_{s}\right), \quad i=1,2 .
\end{aligned}
$$

Notice that this problem couples the subdomains $\Omega_{1}$ and $\Omega_{2}$ through the integral on the boundary $\Gamma_{1,2}$ in (4.11).

The following result establishes a lower bound for the solutions of the problem $\left(\mathrm{AP}_{\lambda}\right)$.

Proposition 4.3. - Under the assumptions (H1)-(H5), let $\left(T_{1}^{\lambda}, T_{2}^{\lambda}, q_{1}^{\lambda}, q_{2}^{\lambda}\right)$ be a solution of $\left(A P_{\lambda}\right)$, then $T_{i}^{\lambda} \geq T_{\min }$ a.e. in $\Omega_{i}, i=1,2$. 
Proof. - Let us choose $z_{i}=\left(T_{\min }-T_{i}^{\lambda}\right)^{+}$as a test function in (4.11), we have

$$
\begin{aligned}
& -\sum_{i=1}^{2} \int_{\Omega_{i}} k_{i}\left(T_{i}^{\lambda}\right)\left|\nabla\left(T_{m i n}-T_{i}^{\lambda}\right)^{+}\right|^{2} d x \\
& +\sum_{i=1}^{2} \int_{\Gamma_{1}^{i}} \alpha\left(T_{i}^{\lambda}-T_{c}\right)\left(T_{m i n}-T_{i}^{\lambda}\right)^{+} d \Gamma \\
& +\int_{\Gamma_{1,2}} \frac{\left(T_{1}^{\lambda}-T_{2}^{\lambda}\right)}{\lambda}\left(\left(T_{m i n}-T_{1}^{\lambda}\right)^{+}-\left(T_{m i n}-T_{2}^{\lambda}\right)^{+}\right) d \Gamma \\
& =\sum_{i=1}^{2} \int_{\Gamma_{3}^{i}} h\left(T_{\min }-T_{i}^{\lambda}\right)^{+} d \Gamma,
\end{aligned}
$$

since $q_{i}^{\lambda}=0$ if $T_{i}^{\lambda} \leq T_{\min }$. We distinguish the sets

$$
\begin{aligned}
B & =\Gamma_{1,2} \cap\left[T_{m i n}>T_{1}^{\lambda}\right] \cap\left[T_{\min }>T_{2}^{\lambda}\right], \\
C_{1} & =\Gamma_{1,2} \cap\left[T_{\min }>T_{1}^{\lambda}\right] \cap\left[T_{\min } \leq T_{2}^{\lambda}\right], \\
C_{2} & =\Gamma_{1,2} \cap\left[T_{\min } \leq T_{1}^{\lambda}\right] \cap\left[T_{\min }>T_{2}^{\lambda}\right],
\end{aligned}
$$

where hereafter [.] denotes the set of points verifying the condition into brackets. The third integral of the left hand side in (4.13) verifies

$$
\begin{aligned}
& \int_{\Gamma_{1,2}} \frac{\left(T_{1}^{\lambda}-T_{2}^{\lambda}\right)}{\lambda}\left(\left(T_{\min }-T_{1}^{\lambda}\right)^{+}-\left(T_{\min }-T_{2}^{\lambda}\right)^{+}\right) d \Gamma \\
& =-\int_{B} \frac{\left(T_{1}^{\lambda}-T_{2}^{\lambda}\right)^{2}}{\lambda} d \Gamma \\
& +\sum_{i=1}^{2} \int_{C_{i}}(-1)^{i+1} \frac{\left(T_{1}^{\lambda}-T_{2}^{\lambda}\right)}{\lambda}\left(T_{\min }-T_{i}^{\lambda}\right)^{+} d \Gamma .
\end{aligned}
$$

Since the right hand side of (4.17) is nonpositive, we deduce the same property for the left hand side of (4.13) while its right hand side is nonnegative. Therefore, $\left(T_{\min }-T_{i}^{\lambda}\right)^{+}=0$ on $\Gamma_{3}^{i}, i=1,2$, and applying the Poincaré's inequality we obtain the result.

\subsection{An equivalent problem}

We are now concerned with the existence of solutions of problem $\left(\operatorname{AP}_{\lambda}\right)$. Let us consider the Kirchhoff transformation given in each domain $\Omega_{i}$ by Vol. $15, n^{\circ} 4-1998$ 
the function $\beta_{i}:\left[T_{\text {min }},+\infty\right) \rightarrow \mathbb{R}$, defined by

$$
\beta_{i}(t)=\int_{0}^{t} k_{i}(s) d s .
$$

We define $U_{i}^{\lambda}$ as the function

$$
U_{i}^{\lambda}=\beta_{i} \circ T_{i}^{\lambda}
$$

and the constants

$$
\begin{aligned}
U_{i, \min } & =\beta_{i}\left(T_{\min }\right), \\
U_{i, s} & =\beta_{i}\left(T_{s}\right),
\end{aligned}
$$

$i=1,2$. Notice that, in general, $U_{1, s}$ is not equal to $U_{2, s}$.

The proof of the following lemma is easy to obtain and is given in [15].

LEMMA 4.1. - i) Both $\beta_{i}$ and $\beta_{i}^{-1}$ are increasing differentiable functions, $i=1,2$.

ii) $\beta_{i}^{-1}(t)$ satisfies a Lipschitz condition with constant $\frac{1}{k_{\min }}$.

iii) $\left(\beta_{i}^{-1}(t)-\beta_{i}^{-1}(\tilde{t})\right)(t-\tilde{t}) \geq \frac{1}{k_{\max }}(t-\tilde{t})^{2}$.

We state the following

\section{- Problem $\left(\widehat{A P}_{\lambda}\right)$}

For a fixed $\lambda>0$, find $\left(U_{1}^{\lambda}, U_{2}^{\lambda}, q_{1}^{\lambda}, q_{2}^{\lambda}\right)$ in $\mathcal{H}^{1}(\Omega) \times L^{\infty}\left(\Omega_{1}\right) \times L^{\infty}\left(\Omega_{2}\right)$ such that

$$
\begin{aligned}
& \sum_{i=1}^{2} \int_{\Omega_{i}} \nabla U_{i}^{\lambda} \cdot \nabla z_{i} d x \\
& +\sum_{i=1}^{2} \int_{\Omega_{i}} h q_{i}^{\lambda} \frac{\partial z_{i}}{\partial x_{1}} d x+\sum_{i=1}^{2} \int_{\Gamma_{1}^{i}} \alpha\left(\beta_{i}^{-1}\left(U_{i}^{\lambda}\right)-T_{c}\right) z_{i} d \Gamma \\
& +\int_{\Gamma_{1,2}} \frac{\left(\beta_{1}^{-1}\left(U_{1}^{\lambda}\right)-\beta_{2}^{-1}\left(U_{2}^{\lambda}\right)\right)}{\lambda}\left(z_{1}-z_{2}\right) d \Gamma \\
& =\sum_{i=1}^{2} \int_{\Gamma_{3}^{i}} h z_{i} d \Gamma, \forall\left(z_{1}, z_{2}\right) \in \mathcal{H}^{1}(\Omega), \\
& q_{i}^{\lambda} \in H\left(U_{i}^{\lambda}-U_{i, s}\right), \quad i=1,2 .
\end{aligned}
$$

PROPOSITION 4.4. - The problem $\left(A P_{\lambda}\right)$ is equivalent to the problem $\left(\widehat{A P}_{\lambda}\right)$. 
Proof. - i) Due to the monotonicity of both $\beta_{i}$ and $\beta_{i}^{-1}$, the following set identities hold

$$
\begin{aligned}
& {\left[T_{i}^{\lambda}<T_{s}\right]=\left[U_{i}^{\lambda}<U_{i, s}\right],} \\
& {\left[T_{i}^{\lambda}>T_{s}\right]=\left[U_{i}^{\lambda}>U_{i, s}\right],} \\
& {\left[T_{i}^{\lambda}=T_{s}\right]=\left[U_{i}^{\lambda}=U_{i, s}\right],}
\end{aligned}
$$

and, consequently, we deduce the equivalence

$$
q_{i}^{\lambda} \in H\left(T_{i}^{\lambda}-T_{s}\right) \Leftrightarrow q_{i}^{\lambda} \in H\left(U_{i}^{\lambda}-U_{i, s}\right), i=1,2
$$

ii) Let $\left(T_{1}^{\lambda}, T_{2}^{\lambda}, q_{1}^{\lambda}, q_{2}^{\lambda}\right)$ be a solution of $\left(\mathrm{AP}_{\lambda}\right)$. Since $\beta_{i}$ is a differentiable function, we have $U_{i}^{\lambda} \in H^{1}\left(\Omega_{i}\right)$ and $\nabla U_{i}^{\lambda}=k_{i}\left(T_{i}^{\lambda}\right) \nabla T_{i}^{\lambda}, i=1,2$ (see [14]). Hence, from (4.11), (4.12) and the equivalence (4.27), we deduce that $\left(U_{1}^{\lambda}, U_{2}^{\lambda}, q_{1}^{\lambda}, q_{2}^{\lambda}\right)$ is a solution of $\left(\widehat{\mathrm{AP}}_{\lambda}\right)$.

Conversely, given $\left(U_{1}^{\lambda}, U_{2}^{\lambda}, q_{1}^{\lambda}, q_{2}^{\lambda}\right)$ a solution of $\left(\widehat{\mathrm{AP}}_{\lambda}\right)$, we define $T_{i}^{\lambda}=\beta_{i}^{-1}\left(U_{i}^{\lambda}\right), i=1,2$. Using that $\beta_{i}^{-1}$ has derivative and lemma $\left.4.1 \mathrm{ii}\right)$, we obtain $T_{i}^{\lambda} \in H^{1}\left(\Omega_{i}\right)$ and $\nabla T_{i}^{\lambda}=\left(k_{i}\left(\beta_{i}^{-1}\left(U_{i}^{\lambda}\right)\right)\right)^{-1} \nabla U_{i}^{\lambda}, i=1,2$. Therefore, from (4.22), (4.23) and the equivalence (4.27), we deduce that $\left(T_{1}^{\lambda}, T_{2}^{\lambda}, q_{1}^{\lambda}, q_{2}^{\lambda}\right)$ is a solution of $\left(\mathrm{AP}_{\lambda}\right)$.

Remark 4.2. - Notice that, given $\left(T_{1}^{\lambda}, T_{2}^{\lambda}\right) \in H^{1}(\Omega)$, the new variable $\left(U_{1}^{\lambda}, U_{2}^{\lambda}\right)$ does not belong, in general, to $H^{1}(\Omega)$.

COROLLARY 4.1. - Under the assumptions (HI)-(H5), let $\left(U_{1}^{\lambda}, U_{2}^{\lambda}, q_{1}^{\lambda}, q_{2}^{\lambda}\right)$ be a solution of problem $\left(\widehat{A P}_{\lambda}\right)$ then

$$
U_{i}^{\lambda} \geq U_{i, \min } \text { a.e. in } \Omega_{i}
$$

where $U_{i, \min }$ is given by (4.20), $i=1,2$.

Proof. - As in the proof of the proposition 4.4, we deduce that $\left(\beta_{1}^{-1}\left(U_{1}^{\lambda}\right), \beta_{2}^{-1}\left(U_{2}^{\lambda}\right), q_{1}^{\lambda}, q_{2}^{\lambda}\right)$ is a solution of $\left(\mathrm{AP}_{\lambda}\right)$. Applying the proposition 4.3 and the monotonicity of $\beta_{i},(4.28)$ is deduced.

\subsection{A penalized problem}

The proof of existence of solution of the problem $\left(\widehat{\mathrm{AP}}_{\lambda}\right)$ goes through the definition of the following regularized problem: 
- Problem $\left(\widehat{\mathbf{A P}}_{\lambda \epsilon}\right)$

For fixed $\lambda>0$ and $\epsilon>0$, find $\left(U_{1 \epsilon}^{\lambda}, U_{2 \epsilon}^{\lambda}\right) \in \mathcal{H}^{1}(\Omega)$ such that

$$
\begin{aligned}
& \sum_{i=1}^{2} \int_{\Omega_{i}} \nabla U_{i \epsilon}^{\lambda} \cdot \nabla z_{i} d x+\sum_{i=1}^{2} \int_{\Omega_{i}} h H_{\epsilon}\left(U_{i \epsilon}^{\lambda}-U_{i, s}\right) \frac{\partial z_{i}}{\partial x_{1}} d x \\
& +\sum_{i=1}^{2} \int_{\Gamma_{1}^{i}} \alpha\left(\beta_{i}^{-1}\left(U_{i \epsilon}^{\lambda}\right)-T_{c}\right) z_{i} d \Gamma \\
& +\int_{\Gamma_{1,2}} \frac{\left(\beta_{1}^{-1}\left(U_{1 \epsilon}^{\lambda}\right)-\beta_{2}^{-1}\left(U_{2 \epsilon}^{\lambda}\right)\right)}{\lambda}\left(z_{1}-z_{2}\right) d \Gamma \\
& =\sum_{i=1}^{2} \int_{\Gamma_{3}^{i}} h z_{i} d \Gamma, \quad \forall\left(z_{1}, z_{2}\right) \in \mathcal{H}^{1}(\Omega),
\end{aligned}
$$

where $U_{i, s}$ is defined by $(4.21), i=1,2$, and

$$
H_{\epsilon}(s)=\left\{\begin{array}{cc}
0 & \text { if } s \leq 0 \\
\frac{s}{c} & \text { if } 0 \leq s \leq \epsilon \\
1 & \text { if } s \geq \epsilon
\end{array}\right.
$$

is the Yosida regularization of the Heaviside multivalued function $\mathrm{H}$.

Let $A_{i}: H^{1}\left(\Omega_{i}\right) \rightarrow H^{1}\left(\Omega_{i}\right)^{\prime}$ be the nonlinear operator defined by

$$
<A_{i}(v), z>=\int_{\Omega_{i}} \nabla v \cdot \nabla z d x+\int_{\Gamma_{1}^{i}} \alpha \beta_{i}^{-1}(v) z d \Gamma+\int_{\Gamma_{1.2}} \frac{\beta_{i}^{-1}(v)}{\lambda} z d \Gamma,
$$

and, for $w \in H^{1}\left(\Omega_{i}\right)$, let $F_{i}(w) \in H^{1}\left(\Omega_{i}\right)^{\prime}$ be given by

$$
\begin{aligned}
<F_{i}(w), z>= & -\int_{\Omega_{i}} h H_{\epsilon}\left(w-U_{i, s}\right) \frac{\partial z}{\partial x_{1}} d x \\
& +\int_{\Gamma_{1}^{i}} \alpha T_{c} z d \Gamma+\int_{\Gamma_{1,2}} \frac{g_{i}}{\lambda} z d \Lambda^{\prime}+\int_{\Gamma_{3}^{i}} h z d \Gamma
\end{aligned}
$$

The idea for proving the existence of problem $\left(\widehat{\mathrm{AP}}_{\lambda_{\epsilon}}\right)$ is to apply the Shauder fixed point theorem to the operator

$$
\begin{aligned}
\mathcal{L}: L^{2}\left(\Gamma_{1,2}\right) \times L^{2}\left(\Gamma_{1,2}\right) & \rightarrow L^{2}\left(\Gamma_{1,2}\right) \times L^{2}\left(\Gamma_{1,2}\right) \\
\left(g_{1}, g_{2}\right) & \rightarrow \mathcal{L}\left(g_{1}, g_{2}\right)=\left(\beta_{2}^{-1}\left(V_{2 \epsilon}^{\lambda}\right), \beta_{1}^{-1}\left(V_{1 \epsilon}^{\lambda}\right)\right)
\end{aligned}
$$

$V_{i \epsilon}^{\lambda}$ being the solution of the following 


\section{- Problem $\left(\widehat{\mathbf{A P}}_{\lambda \epsilon}^{i}\right)$}

For fixed $\lambda>0$ and $\epsilon>0$, and given $g_{i} \in L^{2}\left(\Gamma_{1,2}\right)$, find $V_{i \epsilon}^{\lambda}$ in $H^{1}\left(\Omega_{i}\right)$ such that

$$
<A_{i}\left(V_{i \epsilon}^{\lambda}\right), z_{i}>=<F_{i}\left(V_{i \epsilon}^{\lambda}\right), z_{i}>, \quad \forall z_{i} \in H^{1}\left(\Omega_{i}\right)
$$

for $i=1,2$.

Notice that, in order to define the operator $\mathcal{L}$, two uncoupled problems posed in $\Omega_{1}$ and $\Omega_{2}$ have to be solved, namely $\left(\widehat{\mathrm{AP}}_{\lambda \epsilon}^{i}\right), i=1,2$.

Remark 4.3. - If we set $g_{i}=\beta_{j}^{-1}\left(V_{j \varepsilon}^{\lambda}\right)$ for $j \neq i$ in (4.32), the equation (4.34) becomes (4.29) for the test function $z_{i} \in H^{1}\left(\Omega_{i}\right)$ and $z_{j}=0 \in H^{1}\left(\Omega_{j}\right)$

We prove first that $\mathcal{L}$ is well defined:

Proposition 4.5. - Under the assumptions (HI)-(H5), there exists a solution $V_{i \epsilon}^{\lambda}$ of problem $\left(\widehat{A P}_{\lambda \epsilon}^{i}\right), i=1,2$.

Proof. - We consider $L_{i}$ the mapping which associates to $w \in H^{1}\left(\Omega_{i}\right)$ the solution of the nonlinear problem:

$$
<A_{i}\left(W_{i \epsilon}^{\lambda}\right), z_{i}>=<F_{i}(w), z_{i}>, \quad \forall z_{i} \in H^{1}\left(\Omega_{i}\right)
$$

Step $1 .-A_{i}$ verifies the following properties:

- $A_{i}$ is a continuous operator:

Applying the Cauchy-Schwarz inequality, the lemma 4.1 , ii) and the continuity of the trace, we deduce

$$
\left|<A_{i}\left(v_{n}\right)-A_{i}(v), z_{i}>\right| \leq K\left\|v_{n}-v\right\|_{1,2, \Omega_{i}}\left\|z_{i}\right\|_{1,2, \Omega_{i}},
$$

where $K$ is a constant depending on $\|\alpha\|_{\infty, \Gamma_{1}^{+}}, k_{\text {min }}$ and $\lambda$.

- $A_{i}$ is a strongly monotone operator:

Using lemma 4.1 , iii), we have

$$
\begin{aligned}
<A_{i}\left(v_{1}\right)-A_{i}\left(v_{2}\right), v_{1}-v_{2}> & \geq \int_{\Omega_{i}}\left|\nabla\left(v_{1}-v_{2}\right)\right|^{2} d x \\
+\frac{\alpha_{\min }}{k_{\max }} \int_{\Gamma_{1}^{i}}\left(v_{1}-v_{2}\right)^{2} d \Gamma & +\frac{1}{\lambda k_{\max }} \int_{\Gamma_{1,2}}\left(v_{1}-v_{2}\right)^{2} d \Gamma \\
& \geq C_{i}^{\star}\left\|v_{1}-v_{2}\right\|_{1,2, \Omega_{i}}^{2},
\end{aligned}
$$

with $C_{i}^{\star}$ depending on $\alpha_{\min }, k_{\max }$ and $\lambda$. 
- $A_{i}$ is a coercive operator:

Choosing $v_{2}=0$ in (4.37) and taking into account that $\Lambda_{i}(0)=0$, we deduce

$$
<A_{i}\left(v_{1}\right), v_{1}>\geq C_{i}^{\star}\left\|v_{1}\right\|_{1,2, \Omega_{i}}^{2},
$$

and then

$$
\lim _{\left\|v_{1}\right\|_{1,2, \Omega_{i} \rightarrow \infty} \rightarrow \infty} \frac{<A_{i}\left(v_{1}\right), v_{1}>}{\left\|v_{1}\right\|_{1,2, \Omega_{i}}}=+\infty .
$$

Since $F_{i}(w) \in H^{1}\left(\Omega_{i}\right)^{\prime}$, by applying the Minty-Browder theorem (see [7]), we obtain the existence of a unique $W_{i \epsilon}^{\lambda} \in H^{1}\left(\Omega_{i}\right)$ such that $A_{i}\left(W_{i \epsilon}^{\lambda}\right)=F_{i}(w)$. Therefore, $L_{i}$ is well defined.

Step 2. $-L_{i}$ is compact.

Indeed, it is enough to prove the complete continuity of $L_{i}$. For this purpose, let $\left\{w_{n}\right\}$ be a sequence in $H^{1}\left(\Omega_{i}\right)$ which converges weakly to $w \in H^{1}\left(\Omega_{i}\right)$.

Let $\left\{W_{i \epsilon}^{\lambda n}\right\}$ be the sequence defined by

$$
W_{i \epsilon}^{\lambda n}=L_{i}\left(w_{n}\right), \quad \forall n \in \mathbb{N} .
$$

Then, $W_{i \epsilon}^{\lambda n}$ y $W_{i f}^{\lambda}$ are the unique solutions of

$$
<A_{i}\left(W_{i \epsilon}^{\lambda n}\right), z_{i}>=<F_{i}\left(w_{n}\right), z_{i}>, \forall z_{i} \in H^{1}\left(\Omega_{i}\right),
$$

and

$$
\left.<A_{i}\left(W_{i \epsilon}^{\lambda}\right), z_{i}\right\rangle=\left\langle F_{i}(w), z_{i}\right\rangle, \quad \forall z_{i} \in H^{1}\left(\Omega_{i}\right) .
$$

respectively. By substracting (4.42) from (4.41), taking $z_{i}=W_{i \epsilon}^{\lambda n}-W_{i \epsilon}^{\lambda}$ as a test function, and applying the definition of $A_{i}$ (see (4.31)), we deduce

$$
\begin{aligned}
& <A_{i}\left(W_{i \epsilon}^{\lambda n}\right)-A_{i}\left(W_{i \epsilon}^{\lambda}\right), W_{i \epsilon}^{\lambda n}-W_{i \epsilon}^{\lambda}> \\
& =-\int_{\Omega_{i}} h\left(H_{\epsilon}\left(w_{n}-U_{i, s}\right)-H_{\epsilon}\left(w-U_{i, s}\right)\right) \frac{\partial\left(W_{i \epsilon}^{\lambda n}-W_{i \epsilon}^{\lambda}\right)}{\partial x_{1}} d x .
\end{aligned}
$$

From (4.37), the Cauchy-Schwarz inequality and the Lipschitz continuity of $H_{\epsilon}$, it follows that

$$
C_{i}^{\star}\left\|W_{i \epsilon}^{\lambda n}-W_{i \epsilon}^{\lambda}\right\|_{1,2, \Omega_{i}} \leq \frac{\|h\|_{\infty, \Omega_{i}}}{\epsilon}\left\|w_{n}-w\right\|_{0,2, \Omega_{i}} .
$$

Since $H^{1}\left(\Omega_{i}\right)$ is compactly imbedded in $L^{2}\left(\Omega_{i}\right)$, the complete continuity of $L_{i}$ is now clear. 
Step 3. $-L_{i}$ maps $H^{1}\left(\Omega_{i}\right)$ in a ball.

Indeed, by taking $z_{i}=W_{i \epsilon}^{\lambda}$ as a test function in (4.35) and applying the definition of $A_{i}$ we have

$$
<A_{i}\left(W_{i \epsilon}^{\lambda}\right), W_{i \epsilon}^{\lambda}>=<F_{i}(w), W_{i \epsilon}^{\lambda}>.
$$

Using (4.38), the Cauchy-Schwarz inequality, the fact that $\left|H_{\epsilon}(s)\right| \leq 1$ and the continuity of the trace we deduce

$$
\begin{aligned}
C_{i}^{\star}\left\|W_{i \epsilon}^{\lambda}\right\|_{1,2, \Omega_{i}}^{2} & \leq\|h\|_{0,2, \Omega_{i}}\left\|\frac{\partial W_{i \epsilon}^{\lambda}}{\partial x_{1}}\right\|_{0,2, \Omega_{i}} \\
& +\|\alpha\|_{\infty, \Gamma_{1}^{i}}\left\|T_{c}\right\|_{0,2, \Gamma_{1}^{i}}\left\|W_{i \epsilon}^{\lambda}\right\|_{0,2, \Gamma_{1}^{i}} \\
& +\frac{1}{\lambda}\left\|g_{i}\right\|_{0,2, \Gamma_{1,2}}\left\|W_{i \epsilon}^{\lambda}\right\|_{0,2, \Gamma_{1,2}}+\|h\|_{0,2, \Gamma_{3}^{i}}\left\|W_{i \epsilon}^{\lambda}\right\|_{0,2, \Gamma_{3}^{i}} \\
& \leq \widehat{C}\left\|W_{i \epsilon}^{\lambda}\right\|_{1,2, \Omega_{i}}
\end{aligned}
$$

therefore $W_{i \epsilon}^{\lambda}$ belongs to the ball of $H^{1}\left(\Omega_{i}\right)$ with center 0 and radius $R=\frac{\widehat{C}}{C^{\star}}$.

Finally, the existence of a function $V_{i \epsilon}^{\lambda}$ satisfying (4.34) results from the Schauder fixed point theorem.

The proof of the following result is similar to that obtained in [8], [9]. The difference comes from the boundary integrals of (4.34).

Proposition 4.6. - Under the assumptions (H1)-(H5), the solution $V_{i \epsilon}^{\lambda}$ of the problem $\left(\widehat{A P}_{\lambda \epsilon}^{i}\right)$ is unique, $i=1,2$.

Proof. - Let $V_{i \epsilon}^{\lambda 1}$ and $V_{i \epsilon}^{\lambda 2}$ be two solutions of (4.34) and $Q_{i \epsilon}^{\lambda}=$ $V_{i \epsilon}^{\lambda 1}-V_{i \epsilon}^{\lambda 2}$. We consider the function

$$
p_{\delta}(x)=\left\{\begin{array}{ccc}
\left(1-\frac{\delta}{x}\right)^{+} & \text {si } & x>0 \\
0 & \text { si } & x \leq 0
\end{array}\right.
$$

for a fixed $\delta>0$. Since $p_{\delta}$ is a Lipschitz function, $p_{\delta}\left(Q_{i \epsilon}^{\lambda}\right)$ belongs to $H^{1}\left(\Omega_{i}\right)$ (see [14]). From the equalities satisfied by $V_{i \epsilon}^{\lambda 1}$ and $V_{i \epsilon}^{\lambda 2}$ and taking $z_{i}=p_{\delta}\left(Q_{i \epsilon}^{\lambda}\right)$ as a test function, we obtain

$$
\begin{aligned}
& \delta \int_{\Omega_{i}} \frac{\left|\nabla\left(Q_{i \epsilon}^{\lambda}-\delta\right)^{+}\right|^{2}}{\left(Q_{i \epsilon}^{\lambda}\right)^{2}} d x \\
& +\delta \int_{\Omega_{i}} h\left\{H_{\epsilon}\left(V_{i \epsilon}^{\lambda 1}-U_{i, s}\right)-H_{\epsilon}\left(V_{i \epsilon}^{\lambda 2}-U_{i, s}\right)\right\} \frac{\frac{\partial\left(Q_{i \epsilon}^{\lambda}-\delta\right)^{+}}{\partial x_{1}}}{\left(Q_{i \epsilon}^{\lambda}\right)^{2}} d x
\end{aligned}
$$

Vol. $15, n^{\circ} 4-1998$. 


$$
\begin{aligned}
& +\int_{\Gamma_{1}^{i}} \alpha\left(\beta_{i}^{-1}\left(V_{i \epsilon}^{\lambda 1}\right)-\beta_{i}^{-1}\left(V_{i \epsilon}^{\lambda 2}\right)\right) \frac{\left(Q_{i \epsilon}^{\lambda}-\delta\right)^{+}}{Q_{i \epsilon}^{\lambda}} d \Gamma \\
& +\int_{\Gamma_{1,2}} \frac{\left(\beta_{i}^{-1}\left(V_{i \epsilon}^{\lambda 1}\right)-\beta_{i}^{-1}\left(V_{i \epsilon}^{\lambda 2}\right)\right)}{\lambda} \frac{\left(Q_{i \epsilon}^{\lambda}-\delta\right)^{+}}{Q_{i \epsilon}^{\lambda}} d \Gamma=0 .
\end{aligned}
$$

Taking into account the monotonicity of $\beta_{i}^{-1}$, the lemma 4.1 , iii) and the fact that $h$ is a nonnegative function, we deduce

$$
\begin{aligned}
& \delta \int_{\Omega_{i}} \frac{\left|\nabla\left(Q_{i \epsilon}^{\lambda}-\delta\right)^{+}\right|^{2}}{\left(Q_{i \epsilon}^{\lambda}\right)^{2}} d x+\frac{\alpha_{\min }}{k_{\max }} \int_{\Gamma_{1}^{i}} \frac{\left|\left(Q_{i \epsilon}^{\lambda}-\delta\right)^{+}\right|^{2}}{Q_{i \epsilon}^{\lambda}} d \Gamma \\
& +\frac{1}{\lambda k_{\max }} \int_{\Gamma_{1,2}} \frac{\left|\left(Q_{i \epsilon}^{\lambda}-\delta\right)^{+}\right|^{2}}{Q_{i \epsilon}^{\lambda}} d \Gamma \\
& \leq \delta \int_{\Omega_{i}} h\left|H_{\epsilon}\left(V_{i \epsilon}^{\lambda 1}-U_{i, s}\right)-H_{\epsilon}\left(V_{i \epsilon}^{\lambda 2}-U_{i, s}\right)\right| \frac{\frac{\partial\left(Q_{i \epsilon}^{\lambda}-\delta\right)^{+}}{\partial x_{1}}}{\left(Q_{i \epsilon}^{\lambda}\right)^{2}} \mid d x
\end{aligned}
$$

Since $H_{\epsilon}$ is a Lipschitz function, by using the Cauchy-Schwarz inequality we have

$$
\begin{aligned}
& \delta \int_{\Omega_{i}} \frac{\left|\nabla\left(Q_{i \epsilon}^{\lambda}-\delta\right)^{+}\right|^{2}}{\left(Q_{i \epsilon}^{\lambda}\right)^{2}} d x+\frac{\alpha_{\min }}{k_{\max }} \int_{\Gamma_{1}^{i}} \frac{\left|\left(Q_{i \epsilon}^{\lambda}-\delta\right)^{+}\right|^{2}}{Q_{i \epsilon}^{\lambda}} d \Gamma \\
& +\frac{1}{\lambda k_{\max }} \int_{\Gamma_{1,2}} \frac{\left|\left(Q_{i \epsilon}^{\lambda}-\delta\right)^{+}\right|^{2}}{Q_{i \epsilon}^{\lambda}} d \Gamma \\
& \leq \frac{\delta\|h\|_{0,2, \Omega_{i}}}{c}\left|\frac{\left|\nabla\left(Q_{i \epsilon}^{\lambda}-\delta\right)^{+}\right|}{Q_{i \epsilon}^{\lambda}}\right|_{0,2, \Omega_{i}} .
\end{aligned}
$$

This leads to

$$
\left\|\frac{\mid \nabla\left(Q_{i \epsilon}^{\lambda}-\delta\right)^{+} \|}{Q_{i \epsilon}^{\lambda}}\right\|_{0,2, \Omega_{i}} \leq \frac{\|h\|_{0,2, \Omega_{i}}}{\epsilon}=C_{\epsilon}
$$

with $C_{\epsilon}$ independent of $\delta$. Thus, dividing (4.50) by $\delta$ and using the latter expression, we have

$$
\begin{aligned}
& \int_{\Omega_{i}} \frac{\left|\nabla\left(Q_{i \epsilon}^{\lambda}-\delta\right)^{+}\right|^{2}}{\left(Q_{i \epsilon}^{\lambda}\right)^{2}} d x+\frac{\alpha_{\min }}{\delta k_{\max }} \int_{\Gamma_{1}^{i}} \frac{\left|\left(Q_{i \epsilon}^{\lambda}-\delta\right)^{+}\right|^{2}}{Q_{i \epsilon}^{\lambda}} d \Gamma \\
& +\frac{1}{\delta \lambda k_{\max }} \int_{\Gamma_{1,2}} \frac{\left|\left(Q_{i \epsilon}^{\lambda}-\delta\right)^{+}\right|^{2}}{Q_{i \epsilon}^{\lambda}} d \Gamma \leq C_{\epsilon}^{2} .
\end{aligned}
$$


By passing to the limit when $\delta$ goes to zero, we obtain that $\left(Q_{i \epsilon}^{\lambda}-\delta\right)^{+}=0$ a.e. on $\Gamma_{1}^{i}$ and $\Gamma_{1,2}$. On the other hand, after an easy computation we deduce

$$
\nabla \ln \left(1+\frac{\left(Q_{i \epsilon}^{\lambda}-\delta\right)^{+}}{\delta}\right)=\frac{\nabla\left(Q_{i \epsilon}^{\lambda}-\delta\right)^{+}}{Q_{i \epsilon}^{\lambda}}
$$

and from (4.52) we obtain

$$
\int_{\Omega_{i}}\left|\nabla \ln \left(1+\frac{\left(Q_{i \epsilon}^{\lambda}-\delta\right)^{+}}{\delta}\right)\right|^{2} d x \leq C_{\epsilon}^{2} .
$$

Now by using the Poincaré's inequality, and letting $\delta \rightarrow 0$, we obtain that $Q_{i \epsilon}^{\lambda} \leq 0$ a.e. in $\Omega_{i}$. Interchanging the roles of $V_{i \epsilon}^{\lambda 1}$ and $V_{i \epsilon}^{\lambda 2}$, we deduce $Q_{i \epsilon}^{\lambda}=0$ a.e. in $\Omega_{i}, i=1,2$ which completes the proof.

We are now able to prove the following

Proposition 4.7. - Let $g_{i} \in L^{2}\left(\Gamma_{1,2}\right)$ be such that $g_{i} \leq T_{s}+\frac{\epsilon}{k_{\min }}$ a.e. on $\Gamma_{1,2}$.

Under the assumptions $(H 1)-(H 6)$, the solution $V_{i \epsilon}^{\lambda}$ of $\left(\widehat{A P}_{\lambda \epsilon}^{i}\right)$ verifies

$$
\beta_{i}^{-1}\left(V_{i \epsilon}^{\lambda}\right) \leq T_{s}+\frac{\epsilon}{k_{\min }} \text { a.e. in } \Omega_{i}, i=1,2 \text {. }
$$

Proof. - Let $P_{i \epsilon}^{\lambda}=\beta_{i}^{-1}\left(V_{i \varepsilon}^{\lambda}\right)$. Taking $z_{i}=\left(P_{i \epsilon}^{\lambda}-T_{s}-\frac{\epsilon}{k_{\min }}\right)^{+}$as a test function in (4.34), we obtain

$$
\begin{aligned}
& \int_{\Omega_{i}} \nabla V_{i \epsilon}^{\lambda} \cdot \nabla\left(P_{i \epsilon}^{\lambda}-T_{s}-\frac{\epsilon}{k_{m i n}}\right)^{+} d x+\int_{\Omega_{i}} h \frac{\partial\left(P_{i \epsilon}^{\lambda}-T_{s}-\frac{\epsilon}{k_{m i n}}\right)^{+}}{\partial x_{1}} d x \\
& +\int_{\Gamma_{1}^{i}} \alpha\left(P_{i \epsilon}^{\lambda}-T_{c}\right)\left(P_{i \epsilon}^{\lambda}-T_{s}-\frac{\epsilon}{k_{\min }}\right)^{+} d \Gamma \\
& +\int_{\Gamma_{1,2}} \frac{\left(P_{i \epsilon}^{\lambda}-g_{i}\right)}{\lambda}\left(P_{i \epsilon}^{\lambda}-T_{s}-\frac{\epsilon}{k_{\min }}\right)^{+} d \Gamma \\
& =\int_{\Gamma_{3}^{i}} h\left(P_{i \epsilon}^{\lambda}-T_{s}-\frac{\epsilon}{k_{\min }}\right)^{+} d \Gamma
\end{aligned}
$$

Notice that $H_{\epsilon}\left(V_{i \epsilon}^{\lambda}-U_{i, s}\right)=1$ a.e. in $\mathcal{N}=\left[P_{i \epsilon}^{\lambda}>T_{s}+\frac{\epsilon}{k_{\min }}\right]$. Indeed, if $\left(x_{1}, x_{2}\right) \in \mathcal{N}$ then $V_{i \epsilon}^{\lambda}\left(x_{1}, x_{2}\right)>\beta_{i}\left(T_{s}+\frac{\epsilon}{k_{m i n}}\right)$, and taking into account (H1), it follows that

$$
V_{i \epsilon}^{\lambda}\left(x_{1}, x_{2}\right)>U_{i, s}+\int_{T_{s}}^{T_{s}+\frac{\epsilon}{k_{m, i n}}} k_{i}(s) d s \geq U_{i, s}+\epsilon .
$$


Applying the Green formula in (4.56) we obtain:

$$
\begin{aligned}
& \int_{\Omega_{i}} k_{i}\left(P_{i \epsilon}^{\lambda}\right)\left|\nabla\left(P_{i \epsilon}^{\lambda}-T_{s}-\frac{\epsilon}{k_{\min }}\right)^{+}\right|^{2} d x \\
& +\int_{\Gamma_{1}^{\prime}}\left(\alpha\left(P_{i \epsilon}^{\lambda}-T_{c}\right)+h n_{1}\right)\left(P_{i \epsilon}^{\lambda}-T_{s}-\frac{\epsilon}{k_{\min }}\right)^{+} d \Gamma \\
& +\int_{\Gamma_{1,2}} \frac{\left(P_{i \epsilon}^{\lambda}-g_{i}\right)}{\lambda}\left(P_{i \epsilon}^{\lambda}-T_{s}-\frac{\epsilon}{k_{\min }}\right)^{+} d \Gamma=0,
\end{aligned}
$$

since $h$ only depends on $x_{2}$.

On the other hand, (H6) leads to

$$
\alpha\left(P_{i \epsilon}^{\lambda}-T_{c}\right)+h n_{1}>0 \text { a.e. on } \Gamma_{1}^{i}, i=1,2 .
$$

Therefore, we deduce that all of the terms in the left hand side of (4.58) are nonnegative, and then all of them must be equal to zero. Thus $P_{i \epsilon}^{\lambda} \leq T_{s}+\frac{\epsilon}{k_{\min }}$ a.e. on $\Gamma_{1}^{i}$, and applying the Poincaré's inequality, the result follows.

COROLlaRY 4.2. - The assumptions being those of the proposition 4.7, the inequality $V_{i \epsilon}^{\lambda} \leq U_{i, s}+\frac{k_{\max } \epsilon}{k_{\min }}$ holds a.e. in $\Omega_{i}, i=1,2$.

Proof. - By using the proposition 4.7 and the monotonicity of $\beta_{i}$ we get

$$
V_{i \epsilon}^{\lambda} \leq \beta_{i}\left(T_{s}+\frac{\epsilon}{k_{\min }}\right) \text { a.e. in } \Omega_{i}
$$

Finally, from the definition of $\beta_{i}$ and $(\mathrm{H} 1)$ we obtain

$$
V_{i \epsilon}^{\lambda} \leq \int_{0}^{T_{s}} k_{i}(s) d s+\int_{T_{s}}^{T_{s}+\frac{\epsilon}{k_{m i n}}} k_{i}(s) d s \leq U_{i, s}+\frac{k_{\text {max }} \epsilon}{k_{\text {min }}}, i=1,2 .
$$

PROPOSITION 4.8. - Under the assumptions (HI)-(H6), there exists a solution $\left(U_{1 \epsilon}^{\lambda}, U_{2 \epsilon}^{\lambda}\right)$ of the coupled regularized problem $\left(\widehat{A P}_{\lambda_{\epsilon}}\right)$, defined by (4.29), such that $U_{i \epsilon}^{\lambda} \leq U_{i, s}+\frac{k_{\text {max }}}{k_{\text {min }}}$ e a.e. in $\Omega_{i}, i=1,2$.

Proof. - Let us consider the space $L^{2}\left(\Gamma_{1,2}\right) \times L^{2}\left(\Gamma_{1,2}\right)$ with the norm

$$
\|(f, g)\|_{L^{2}\left(\Gamma_{1,2}\right) \times L^{2}\left(\Gamma_{1,2}\right)}=\left(\|f\|_{0,2, \Gamma_{1,2}}^{2}+\|g\|_{0,2, \Gamma_{1,2}}^{2}\right)^{\frac{1}{2}},
$$


and $\mathcal{L}$ the operator introduced in (4.33), where $V_{i \epsilon}^{\lambda}$ is the solution of problem ( $\widehat{\mathrm{AP}}_{\lambda \epsilon}^{i}$ ) corresponding to $g_{i}, i=1,2$. The propositions (4.5) and (4.6) imply that $\mathcal{L}$ is well defined.

Let $\left\{\left(g_{1}^{n}, g_{2}^{n}\right)\right\}$ be a bounded sequence in $L^{2}\left(\Gamma_{1,2}\right) \times L^{2}\left(\Gamma_{1,2}\right)$. Then, $\left\{g_{i}^{n}\right\}$ is a bounded sequence in $L^{2}\left(\Gamma_{1,2}\right), i=1,2$.

We denote by $V_{i \epsilon}^{\lambda n}$ the solution of $\left(\widehat{\mathrm{AP}}_{\lambda \epsilon}^{i}\right)$ corresponding to $g_{i}^{n}, i=1,2$. If we set $z_{i}=V_{i \epsilon}^{\lambda n}$ as a test function in (4.34), we have

$$
<A_{i}\left(V_{i \epsilon}^{\lambda n}\right), V_{i \epsilon}^{\lambda n}>=<F_{i}\left(V_{i \epsilon}^{\lambda n}\right), V_{i \epsilon}^{\lambda n}>\text {. }
$$

Applying (4.38), the Cauchy-Schwarz inequality and $\left|H_{\epsilon}(s)\right| \leq 1$, it follows that

$$
\begin{aligned}
& C_{i}^{\star}\left\|V_{i \epsilon}^{\lambda n}\right\|_{1,2, \Omega_{i}}^{2} \leq\|h\|_{0,2, \Gamma_{3}^{i}}\left\|V_{i \epsilon}^{\lambda n}\right\|_{0,2, \Gamma_{3}^{i}} \\
& +\|h\|_{0,2, \Omega_{i}}\left\|\frac{\partial V_{i \epsilon}^{\lambda n}}{\partial x_{1}}\right\|_{0,2, \Omega_{i}}+\|\alpha\|_{\infty, \Gamma_{1}^{i}}\left\|T_{c}\right\|_{0,2, \Gamma_{1}^{i}}\left\|V_{i \epsilon}^{\lambda n}\right\|_{0,2, \Gamma_{1}^{i}} \\
& +\frac{1}{\lambda}\left\|g_{i}^{n}\right\|_{0,2, \Gamma_{1,2}}\left\|V_{i \epsilon}^{\lambda n}\right\|_{0,2, \Gamma_{1,2}} \leq \widehat{C}\left\|V_{i \epsilon}^{\lambda n}\right\|_{1,2, \Omega_{i}}
\end{aligned}
$$

hence,

$$
\left\|V_{i \epsilon}^{\lambda n}\right\|_{1,2, \Omega_{i}} \leq \tilde{C}
$$

where $\tilde{C}$ is a constant which depends on $\lambda$ but not on $\epsilon$. It follows that $\left\{V_{i \epsilon}^{\lambda n}\right\}$ is bounded in $H^{1}\left(\Omega_{i}\right)$, and then it has a subsequence $\left\{V_{i \epsilon}^{\lambda n_{k}}\right\}$ weakly convergent to an element $V_{i \mathrm{f}}^{\lambda}$ in $H^{1}\left(\Omega_{i}\right), i=1,2$. Consequently, $\left\{V_{i \epsilon}^{\lambda n_{k}}\right\}$ converges strongly to $V_{i \epsilon}^{\lambda}$ in $L^{2}\left(\Gamma_{1,2}\right)$. Hence $\beta_{i}^{-1}$ being Lipschitz continuous it follows that $\left\{\beta_{i}^{-1}\left(V_{i \epsilon}^{\lambda n_{k}}\right)\right\}$ converges strongly to $\beta_{i}^{-1}\left(V_{i f}^{\lambda}\right)$ in $L^{2}\left(\Gamma_{1,2}\right), i=1,2$. Thus $\mathcal{L}$ is compact.

We define

$$
\begin{aligned}
\mathcal{M}=\left\{\left(g_{1}, g_{2}\right) \in L^{2}\left(\Gamma_{1,2}\right) \times L^{2}\left(\Gamma_{1,2}\right):\right. \\
\left.\quad 0 \leq g_{i} \leq T_{s}+\frac{\epsilon}{k_{\text {min }}} \text { a.e. in } \Gamma_{1,2}, i=1,2\right\}
\end{aligned}
$$

It is clear from proposition 4.7 that

$$
\mathcal{L}(\mathcal{M}) \subset \mathcal{M}
$$

$\mathcal{M}$ being a closed bounded convex set, the existence of a fixed point of $\mathcal{L}$, denoted by $\left(U_{1 \epsilon}^{\lambda}, U_{2 \epsilon}^{\lambda}\right)$, results from the Schauder fixed point theorem.

Vol. $15, n^{4}$ 4-1998. 
Finally, from corollary 4.2 we obtain

$$
U_{i \epsilon}^{\lambda} \leq U_{i, 4}+\frac{k_{\text {max }}}{k_{\text {min }}} \text { e a.e. in } \Omega_{i}, i=1,2 .
$$

In the next proposition we pass to the limit in $\epsilon$.

Proposition 4.9. - Under the assumptions (HI)-(H6), there exists a solution $\left(U_{1}^{\lambda}, U_{2}^{\lambda}, q_{1}^{\lambda}, q_{2}^{\lambda}\right)$ of the problem $\left(\widehat{A P}_{\lambda}\right)$, defined by (4.22) and (4.23), such that $U_{i}^{\lambda} \leq U_{i, s}$ a.e. in $\Omega_{i}, i=1,2$.

Proof. - For a fixed $\epsilon>0$, let $\left(U_{1 \epsilon}^{\lambda}, U_{2 \epsilon}^{\lambda}\right)$ be a solution of $\left(\widehat{\mathrm{AP}}_{\lambda \epsilon}\right)$.

As in the proof of the proposition 4.8 , we deduce that $\left\{\left(U_{1 \epsilon}^{\lambda}, U_{2 \epsilon}^{\lambda}\right)\right\}$ is bounded in $\mathcal{H}^{1}(\Omega)$ independently of $\epsilon$ and so we can extract a subsequence of $\epsilon$ still denoted by $\epsilon$ such that

$$
\begin{aligned}
& \left\{U_{i \epsilon}^{\lambda}\right\} \rightarrow U_{i}^{\lambda} \text { in } H^{1}\left(\Omega_{i}\right) \text { weakly, } \\
& \left\{U_{i \epsilon}^{\lambda}\right\} \rightarrow U_{i}^{\lambda} \text { in } L^{2}\left(\Omega_{i}\right) \text { strongly, } \\
& \left\{U_{i \epsilon}^{\lambda}\right\} \rightarrow U_{i}^{\lambda} \text { a.e. in } \Omega_{i} .
\end{aligned}
$$

Moreover, $\left\{U_{i \epsilon}^{\lambda}\right\}$ converges strongly to $U_{i}^{\lambda}$ in $L^{2}\left(\Gamma_{1,2}\right), L^{2}\left(\Gamma_{1}^{i}\right)$ and $L^{2}\left(\Gamma_{3}^{i}\right)$, $i-1,2$. From (4.68) and (4.71) it follows that

$$
U_{i}^{\lambda}(x) \leq U_{i, s} \text { a.e. in } \Omega_{i}, i=1,2 .
$$

Since $\left\{H_{\epsilon}\left(U_{1 \epsilon}^{\lambda}-U_{1, s}\right), H_{\epsilon}\left(U_{2 \epsilon}^{\lambda}-U_{2, s}\right)\right\}$ is bounded in $L^{2}\left(\Omega_{1}\right) \times L^{2}\left(\Omega_{2}\right)$, there exists $\left(q_{1}^{\lambda}, q_{2}^{\lambda}\right)$ in $L^{2}\left(\Omega_{1}\right) \times L^{2}\left(\Omega_{2}\right)$ such that

$$
\left\{H_{\epsilon}\left(U_{i \epsilon}^{\lambda}-U_{i, s}\right)\right\} \rightarrow q_{i}^{\lambda} \text { in } L^{2}\left(\Omega_{i}\right), i=1,2 .
$$

On the other hand, notice that $q_{i}^{\lambda}$ belongs to the closed convex set $\mathcal{N}_{i}$ defined by

$$
\mathcal{N}_{i}=\left\{f \in L^{\infty}\left(\Omega_{i}\right): 0 \leq f \leq 1 \text { a.e. in } \Omega_{i}\right\}
$$

since this set is weakly closed.

Finally, in the set $\left[U_{i}^{\lambda}<U_{i, s}\right]$ we have

$$
H_{\epsilon}\left(U_{i \epsilon}^{\lambda}-U_{i, s}\right) \rightarrow 0 \text { a.e., }
$$

and applying the Lebesgue theorem we get

$$
H_{\epsilon}\left(U_{i \epsilon}^{\lambda}-U_{i, s}\right) \rightarrow 0 \text { in } L^{2}\left(\left[U_{i}^{\lambda}<U_{i, s}\right]\right) .
$$

From (4.73), we deduce

$$
H_{\epsilon}\left(U_{i \epsilon}^{\lambda}-U_{i, s}\right) \rightarrow q_{i}^{\lambda} \text { in } L^{2}\left(\left[U_{i}^{\lambda}<U_{i, s}\right]\right),
$$

and by the uniqueness of the limit

$$
q_{i}^{\lambda}=0 \text { a.e in }\left[U_{i}^{\lambda}<U_{i, s}\right], i=1,2,
$$

which completes the proof. 


\section{A PRIORI ESTIMATES}

This section is devoted to obtaining some estimates for the solution of the auxiliary problem $\left(\widehat{\mathrm{AP}}_{\lambda}\right)$.

PROPOSITION 5.10. - Under the assumptions (H1)-(H6), a solution $\left(U_{1}^{\lambda}, U_{2}^{\lambda}, q_{1}^{\lambda}, q_{2}^{\lambda}\right)$ of the problem $\left(\widehat{A P}_{\lambda}\right)$, defined by (4.22) and (4.23), satisfies

$$
U_{i}^{\lambda} \leq U_{i, s} \text { a.e. in } \Omega_{i}, i=1,2 .
$$

Proof. - Taking $\left(z_{1}, z_{2}\right)=\left(\left(\beta_{1}^{-1}\left(U_{1}^{\lambda}\right)-T_{s}\right)^{+},\left(\beta_{2}^{-1}\left(U_{2}^{\lambda}\right)-T_{s}\right)^{+}\right)$as a test function in (4.22) and using $\nabla U_{i}^{\lambda}=k_{i}\left(\beta_{i}^{-1}\left(U_{i}^{\lambda}\right)\right) \nabla \beta_{i}^{-1}\left(U_{i}^{\lambda}\right)$, we obtain

$$
\begin{aligned}
& \sum_{i=1}^{2} \int_{\Omega_{i}} k_{i}\left(\beta_{i}^{-1}\left(U_{i}^{\lambda}\right)\right)\left|\nabla\left(\beta_{i}^{-1}\left(U_{i}^{\lambda}\right)-T_{s}\right)^{+}\right|^{2} d x \\
& +\sum_{i=1}^{2} \int_{\Omega_{i}} h q_{i}^{\lambda} \frac{\partial\left(\beta_{i}^{-1}\left(U_{i}^{\lambda}\right)-T_{s}\right)^{+}}{\partial x_{1}} d x \\
& +\sum_{i=1}^{2} \int_{\Gamma_{1}^{i}} \alpha\left(\beta_{i}^{-1}\left(U_{i}^{\lambda}\right)-T_{c}\right)\left(\beta_{i}^{-1}\left(U_{i}^{\lambda}\right)-T_{s}\right)^{+} d \Gamma \\
& +\int_{\Gamma_{1,2}} \frac{\left(\beta_{1}^{-1}\left(U_{1}^{\lambda}\right)-\beta_{2}^{-1}\left(U_{2}^{\lambda}\right)\right)}{\lambda}\left(\left(\beta_{1}^{-1}\left(U_{1}^{\lambda}\right)-T_{s}\right)^{+}-\left(\beta_{2}^{-1}\left(U_{2}^{\lambda}\right)-T_{s}\right)^{+}\right) d \Gamma \\
& =\sum_{i=1}^{2} \int_{\Gamma_{3}^{i}} h\left(\beta_{i}^{-1}\left(U_{i}^{\lambda}\right)-T_{s}\right)^{+} d \Gamma .
\end{aligned}
$$

Notice that $q_{i}^{\lambda}=1$ a.e. in $\left[U_{i}^{\lambda}>U_{i, s}\right]$. By applying the Green formula and taking into account that $h$ only depends on $x_{2}$ it follows that

$$
\begin{aligned}
& \sum_{i=1}^{2} \int_{\Omega_{i}} k_{i}\left(\beta_{i}^{-1}\left(U_{i}^{\lambda}\right)\right)\left|\nabla\left(\beta_{i}^{-1}\left(U_{i}^{\lambda}\right)-T_{s}\right)^{+}\right|^{2} d x \\
& +\sum_{i=1}^{2} \int_{\Gamma_{1}^{i}}\left(\alpha\left(\beta_{i}^{-1}\left(U_{i}^{\lambda}\right)-T_{c}\right)+h n_{1}\right)\left(\beta_{i}^{-1}\left(U_{i}^{\lambda}\right)-T_{s}\right)^{+} d \Gamma \\
& +\int_{\Gamma_{1,2}} \frac{\left(\beta_{1}^{-1}\left(U_{1}^{\lambda}\right)-\beta_{2}^{-1}\left(U_{2}^{\lambda}\right)\right)}{\lambda}\left(\left(\beta_{1}^{-1}\left(U_{1}^{\lambda}\right)-T_{s}\right)^{+}-\left(\beta_{2}^{-1}\left(U_{2}^{\lambda}\right)-T_{s}\right)^{+}\right) d \Gamma=0 .
\end{aligned}
$$

From (H6) we deduce that $\alpha\left(\beta_{i}^{-1}\left(U_{i}^{\lambda}\right)-T_{c}\right)+h n_{1}>0$ a.e. on $\Gamma_{1}^{i}$. On the other hand, as in the proof of the proposition 4.3 , it follows that Vol. $15, \mathrm{n}^{\circ} 4-1998$. 
the third term of the expression (5.3) is nonnegative. Consequently all of the terms of (5.3) are nonnegative and then they are equal to zero. Thus $\left(\beta_{i}^{-1}\left(U_{i}^{\lambda}\right)-T_{s}\right)^{+}=0$ a.e. in $\Gamma_{1}^{i}, i=1,2$. By the Poincaré's inequality and the monotonicity of $\beta_{i}^{-1}, i=1,2$, we deduce $(5.1)$.

COROLlary 5.3. - Under the assumptions (H1)-(H6), a solution $\left(T_{1}^{\lambda}, T_{2}^{\lambda}, q_{1}^{\lambda}, q_{2}^{\lambda}\right)$ of the problem $\left(A P_{\lambda}\right)$ defined by (4.11) and (4.12) verifies

$$
T_{i}^{\lambda} \leq T_{s} \text { a.e. in } \Omega_{i} . i=1,2 .
$$

Proof. - As in the proof of proposition 4.4, we obtain that $\left(U_{1}^{\lambda}, U_{2}^{\lambda}, q_{1}^{\lambda}, q_{2}^{\lambda}\right)$ is a solution of the problem $\left(\widehat{\mathrm{AP}}_{\lambda}\right)$. By applying the proposition 5.10 it follows that $U_{i}^{\lambda} \leq U_{i, k}$ a.e. in $\Omega_{i}$ and then using the monotonicity of $\beta_{i}^{-1}, i=1,2$, we have (5.4).

Proposition 5.11. - Under the assumptions (HI)-(H6), a solution $\left(U_{1}^{\lambda}, U_{2}^{\lambda}, q_{1}^{\lambda}, q_{2}^{\lambda}\right)$ of the problem $\left(\widehat{A P}_{\lambda}\right)$ verifies

$$
\left\|\beta_{1}^{-1}\left(U_{1}^{\lambda}\right)-\beta_{2}^{-1}\left(U_{2}^{\lambda}\right)\right\|_{0,2, \Gamma_{1,2}} \leq K \lambda^{\frac{1}{2}}, \lambda>0
$$

with $K$ a constant which does not depend on $\lambda$.

Proof. - Taking $\left(z_{1}, z_{2}\right)=\left(\beta_{1}^{-1}\left(U_{1}^{\lambda}\right), \beta_{2}^{-1}\left(U_{2}^{\lambda}\right)\right)$ as a test function in (4.22), we obtain

$$
\begin{aligned}
& \sum_{i=1}^{2} \int_{\Omega_{i}} \frac{1}{k_{i}\left(\beta_{i}^{-1}\left(U_{i}^{\lambda}\right)\right)}\left|\nabla U_{i}^{\lambda}\right|^{2} d x+\sum_{i=1}^{2} \int_{\Omega_{i}} \frac{h q_{i}^{\lambda}}{k_{i}\left(\beta_{i}^{-1}\left(U_{i}^{\lambda}\right)\right)} \frac{\partial U_{i}^{\lambda}}{\partial x_{1}} d x \\
& +\sum_{i=1}^{2} \int_{\Gamma_{1}^{i}} \alpha\left(\beta_{i}^{-1}\left(U_{i}^{\lambda}\right)-T_{c}\right) \beta_{i}^{-1}\left(U_{i}^{\lambda}\right) d \Gamma+\int_{\Gamma_{1,2}} \frac{\left(\beta_{1}^{-1}\left(U_{1}^{\lambda}\right)-\beta_{2}^{-1}\left(U_{2}^{\lambda}\right)\right)^{2}}{\lambda} d \Gamma \\
& =\sum_{i=1}^{2} \int_{\Gamma_{3}^{i}} h \beta_{i}^{-1}\left(U_{i}^{\lambda}\right) d \Gamma
\end{aligned}
$$

From proposition 5.10, we have $U_{i}^{\lambda} \leq U_{i, s}$ a.e. in $\Omega_{i}$. Using both $q_{i}^{\lambda}=0$ a.e. in $\left[U_{i}^{\lambda}<U_{i, s}\right]$ and $\frac{\partial U_{i}^{\lambda}}{\partial x_{1}}=0$ a.e. in $\left[U_{i}^{\lambda}=U_{i, s}\right]$, the second term of (5.6) vanishes and we have

$$
\begin{aligned}
& \sum_{i=1}^{2} \int_{\Omega_{i}} \frac{1}{k_{i}\left(\beta_{i}^{-1}\left(U_{i}^{\lambda}\right)\right)}\left|\nabla U_{i}^{\lambda}\right|^{2} d x+\sum_{i=1}^{2} \int_{\Gamma_{1}^{i}} \alpha\left(\beta_{i}^{-1}\left(U_{i}^{\lambda}\right)\right)^{2} d \Gamma \\
& +\int_{\Gamma_{1,2}} \frac{\left(\beta_{1}^{-1}\left(U_{1}^{\lambda}\right)-\beta_{2}^{-1}\left(U_{2}^{\lambda}\right)\right)^{2}}{\lambda} d \Gamma \\
& =\sum_{i=1}^{2} \int_{\Gamma_{3}^{i}} h \beta_{i}^{-1}\left(U_{i}^{\lambda}\right) d \Gamma+\sum_{i=1}^{2} \int_{\Gamma_{1}^{i}} \alpha T_{c} \beta_{i}^{-1}\left(U_{i}^{\lambda}\right) d \Gamma .
\end{aligned}
$$


Since all of the terms on the left hand side of (5.7) are nonnegative, we obtain

$$
\begin{aligned}
& \int_{\Gamma_{1,2}} \frac{\left(\beta_{1}^{-1}\left(U_{1}^{\lambda}\right)-\beta_{2}^{-1}\left(U_{2}^{\lambda}\right)\right)^{2}}{\lambda} d \Gamma \\
& \leq \sum_{i=1}^{2}\left(\int_{I_{3}^{\prime}} h \beta_{i}^{-1}\left(U_{i}^{\lambda}\right) d \Gamma+\int_{\Gamma_{1}^{i}} \alpha T_{c} \beta_{i}^{-1}\left(U_{i}^{\lambda}\right) d \Gamma\right) .
\end{aligned}
$$

By the Cauchy-Schwarz inequality, it follows that

$$
\begin{aligned}
& \int_{\Gamma_{1,2}} \frac{\left(\beta_{1}^{-1}\left(U_{1}^{\lambda}\right)-\beta_{2}^{-1}\left(U_{2}^{\lambda}\right)\right)^{2}}{\lambda} d \Gamma \\
& \leq \sum_{i=1}^{2}\|h\|_{\infty, \Gamma_{3}^{i}}\left\|\beta_{i}^{-1}\left(U_{i}^{\lambda}\right)\right\|_{0,2, \Gamma_{3}^{i}} \operatorname{meas}\left(\Gamma_{3}^{i}\right)^{\frac{1}{2}} \\
& +\sum_{i=1}^{2}\|\alpha\|_{\infty, \Gamma_{1}^{i}}\left\|T_{c}\right\|_{0,2, \Gamma_{1}^{i}}\left\|\beta_{i}^{-1}\left(U_{i}^{\lambda}\right)\right\|_{0,2, \Gamma_{1}^{i}}
\end{aligned}
$$

where here and in the sequel meas(.) stands for the Lebesgue measure of the set in parenthesis.

Let $K$ be the constant given by

$$
\begin{aligned}
K^{2}=T_{s}( & \sum_{i=1}^{2}\|h\|_{\infty, \Gamma_{3}^{i}} \operatorname{meas}\left(\Gamma_{3}^{i}\right) \\
& \left.+\sum_{i=1}^{2}\|\alpha\|_{\infty, \Gamma_{1}^{i}}\left\|T_{c}\right\|_{0,2, \Gamma_{1}^{i}} \operatorname{meas}\left(\Gamma_{1}^{i}\right)^{\frac{1}{2}}\right) .
\end{aligned}
$$

Notice that $K$ is independent of $\lambda$. Thus, from (5.9) and taking into account that $\beta_{i}^{-1}\left(U_{i}^{\lambda}\right) \leq T_{s}$ a.e. on $\Gamma_{3}^{2}$ and $\Gamma_{1}^{i}, i=1,2$, we obtain (5.5).

Remark 5.4. - By definition of $\beta_{i}$ and using ( $\left.\mathrm{H} 1\right)$, it is easy to deduce the following inequality

$$
U_{i}^{\lambda} \leq \beta_{i}^{-1}\left(U_{i}^{\lambda}\right) k_{\max }
$$

Thus, from (5.7), we obtain

$$
\frac{1}{k_{\max }}\left(\sum_{i=1}^{2} \int_{\Omega_{i}}\left|\nabla U_{i}^{\lambda}\right|^{2} d x\right)+\frac{\alpha_{\min }}{k_{\max }^{2}}\left(\sum_{i=1}^{2} \int_{\Gamma_{1}^{i}}\left(U_{i}^{\lambda}\right)^{2} d \Gamma\right) \leq K^{2} \text {. }
$$

Consequently, $\left(U_{1}^{\lambda}, U_{2}^{\lambda}\right)$ is bounded in $\mathcal{H}^{1}(\Omega)$ by a constant which is independent of $\lambda$. 


\section{EXISTENCE OF A SOLUTION}

We are now able to prove the main existence result

Proposition 6.12. - Under the assumptions (HI)-(H6), there exists a solution $\left(T_{1}, T_{2}, q_{1}, q_{2}\right)$ of the problem (WP), defined by (3.25) and (3.26).

Proof. - For a fixed $\lambda>0$, we consider $\left(U_{1}^{\lambda}, U_{2}^{\lambda}, q_{1}^{\lambda}, q_{2}^{\lambda}\right)$ the solution of problem $\left(\widehat{\mathrm{AP}}_{\lambda}\right)$.

From the remark 5.4, $\left\{\left(U_{1}^{\lambda}, U_{2}^{\lambda}\right)\right\}$ is bounded in $\mathcal{H}^{1}(\Omega)$ independently of $\lambda$. Thus, we can extract a subsequence still denoted by $\lambda$ such that

$$
\begin{aligned}
& \left\{U_{i}^{\lambda}\right\} \rightarrow U_{i} \text { in } H^{1}\left(\Omega_{i}\right) \text { weakly, } \\
& \left\{U_{i}^{\lambda}\right\} \rightarrow U_{i} \text { in } L^{2}\left(\Omega_{i}\right) \text { strongly }, \\
& \left\{U_{i}^{\lambda}\right\} \rightarrow U_{i} \text { a.e. in } \Omega_{i}, \\
& \left\{U_{i}^{\lambda}\right\} \rightarrow U_{i} \text { in } L^{2}\left(\Gamma_{1,2}\right) .
\end{aligned}
$$

Furthermore, $\left\{U_{i}^{\lambda}\right\}$ converges to $U_{i}$ strongly in $L^{2}\left(\Gamma_{1}^{i}\right)$ and $L^{2}\left(\Gamma_{3}^{i}\right)$, $i=1,2$. Then, from (6.3), the proposition 5.10 and the corollary 4.1 , we obtain

$$
U_{i, \min } \leq U_{i} \leq U_{i, s} \text { a.e. in } \Omega_{i}, i=1,2 .
$$

Since $\left\{\left(q_{1}^{\lambda}, q_{2}^{\lambda}\right)\right\}$ is bounded in $L^{2}\left(\Omega_{1}\right) \times L^{2}\left(\Omega_{2}\right)$, there exists $\left(q_{1}, q_{2}\right)$ in $L^{2}\left(\Omega_{1}\right) \times L^{2}\left(\Omega_{2}\right)$ such that

$$
\left\{q_{i}^{\lambda}\right\}-q_{i} \text { in } L^{2}\left(\Omega_{i}\right), i=1,2,
$$

and $q_{i}$ belongs to the weakly closed set $\mathcal{N}_{i}$ defined by (4.74). As in the proof of proposition $4.9, q_{i}$ vanishes a.e. in $\left[U_{i}<U_{i, s}\right], i=1,2$. Thus, it follows that

$$
q_{i} \in H\left(U_{i}-U_{i, s}\right), i=1,2 .
$$

On the other hand, if we take $\left(z_{1}, z_{2}\right)$ in $H^{1}(\Omega)$ as a test function in (4.22), we get

$$
\begin{aligned}
\sum_{i=1}^{2} \int_{\Omega_{i}} \nabla U_{i}^{\lambda} \cdot \nabla z_{i} d x & +\sum_{i=1}^{2} \int_{\Omega_{i}} h q_{i}^{\lambda} \frac{\partial z_{i}}{\partial x_{1}} d x \\
& +\sum_{i=1}^{2} \int_{\Gamma_{1}^{i}} \alpha\left(\beta_{i}^{-1}\left(U_{i}^{\lambda}\right)-T_{c}\right) z_{i} d \Gamma \\
& =\sum_{i=1}^{2} \int_{\Gamma_{3}^{i}} h z_{i} d \Gamma, \quad \forall\left(z_{1}, z_{2}\right) \in H^{1}(\Omega)
\end{aligned}
$$


and letting $\lambda \rightarrow 0$, we deduce

$$
\begin{aligned}
\sum_{i=1}^{2} \int_{\Omega_{i}} \nabla U_{i} . \nabla z_{i} d x & +\sum_{i=1}^{2} \int_{\Omega_{i}} h q_{i} \frac{\partial z_{i}}{\partial x_{1}} d x+\sum_{i=1}^{2} \int_{\Gamma_{1}^{i}} \alpha\left(\beta_{i}^{-1}\left(U_{i}\right)-T_{c}\right) z_{i} d \Gamma \\
& =\sum_{i=1}^{2} \int_{\Gamma_{3}^{i}} h z_{i} d \Gamma, \quad \forall\left(z_{1}, z_{2}\right) \in H^{1}(\Omega) .
\end{aligned}
$$

For $i=1,2$, we set $T_{i}=\beta_{i}^{-1}\left(U_{i}\right)$. By taking into account the equivalence (4.27), it follows that

$$
\begin{aligned}
\sum_{i=1}^{2} \int_{\Omega_{i}} k_{i}\left(T_{i}\right) \nabla T_{i} . \nabla z_{i} d x & +\sum_{i=1}^{2} \int_{\Omega_{i}} h q_{i} \frac{\partial z_{i}}{\partial x_{1}} d x+\sum_{i=1}^{2} \int_{\Gamma_{1}^{i}} \alpha\left(T_{i}-T_{c}\right) z_{i} d \Gamma \\
& =\sum_{i=1}^{2} \int_{\Gamma_{3}^{i}} h z_{i} d \Gamma, \quad \forall\left(z_{1}, z_{2}\right) \in H^{1}(\Omega), \quad(6.10) \\
q_{i} & \in H\left(T_{i}-T_{s}\right), \quad i=1,2 .
\end{aligned}
$$

Thus, both $\left(T_{1}, T_{2}\right) \in \mathcal{H}^{1}(\Omega)$ and $\left(q_{1}, q_{2}\right) \in L^{\infty}\left(\Omega_{1}\right) \times L^{\infty}\left(\Omega_{2}\right)$ verify (3.25) and (3.26).

Finally, (5.5) leads to

$$
\left\{\beta_{1}^{-1}\left(U_{1}^{\lambda}\right)-\beta_{2}{ }^{1}\left(U_{2}^{\lambda}\right)\right\} \rightarrow 0 \text { in } L^{2}\left(\Gamma_{1,2}\right) .
$$

On the other hand, taking into account that $\beta_{i}^{-1}$ is a Lipschitz function for $i=1,2$, (6.4) leads to

$$
\left\{\beta_{1}^{-1}\left(U_{1}^{\lambda}\right)-\beta_{2}^{-1}\left(U_{2}^{\lambda}\right)\right\} \rightarrow T_{1}-T_{2} \text { in } L^{2}\left(\Gamma_{1,2}\right) .
$$

Thus

$$
T_{1}=T_{2} \text { in } L^{2}\left(\Gamma_{1,2}\right)
$$

and then $\left(T_{1}, T_{2}\right) \in H^{1}(\Omega)$ which finishes the proof.

From the proof of the proposition 6.12 , we deduce that $T_{i} \leq T_{s}$ a.e. in $\Omega_{i}, i=1,2$. Actually this property holds for every solution of (WP):

Proposition 6.13. - Under the assumptions (H1)-(H6), a solution of the problem (WP) satisfies

$$
T_{i} \leq T_{s} \text { a.e. in } \Omega_{i}, i=1,2 \text {. }
$$

Vol. $15, \mathrm{n}^{\circ} 4-1998$. 
Proof. - Let us choose $z_{i}=\left(T_{i}-T_{s}\right)^{+}$as a test function in (3.25), we have

$$
\begin{aligned}
& \sum_{i=1}^{2} \int_{\Omega_{i}} k\left(T_{i}\right)\left|\nabla\left(T_{i}-T_{s}\right)^{+}\right|^{2} d x+\sum_{i=1}^{2} \int_{\Omega_{i}} h \frac{\partial\left(T_{i}-T_{s}\right)^{+}}{\partial x_{1}} d x \\
& +\sum_{i=1}^{2} \int_{\Gamma_{1}^{i}} \alpha\left(T_{i}-T_{c}\right)\left(T_{i}-T_{s}\right)^{+} d \Gamma=\sum_{i=1}^{2} \int_{\Gamma_{3}^{i}} h\left(T_{i}-T_{s}\right)^{+} d \Gamma
\end{aligned}
$$

since $q_{i}=1$ a.e. in $\left[T_{i}>T_{s}\right], i=1,2$. By applying the Green formula and taking into account that $h$ only depends on $x_{2}$ it follows that

$$
\begin{aligned}
& \sum_{i=1}^{2} \int_{\Omega_{i}} k\left(T_{i}\right)\left|\nabla\left(T_{i}-T_{s}\right)^{+}\right|^{2} d x \\
& +\sum_{i=1}^{2} \int_{\Gamma_{i}^{i}}\left(h n_{1}+\alpha\left(T_{i}-T_{c}\right)\right)\left(T_{i}-T_{s}\right)^{+} d \Gamma=0 .
\end{aligned}
$$

From (H6) we deduce that $h n_{1}+\alpha\left(T_{i}-T_{c}\right)>h n_{1}+\alpha\left(T_{s}-T_{c}\right) \geq 0$ a.e. on $\Gamma_{1}^{i}, i=1,2$. Consequently, all of the terms of (6.17) are nonnegative, and then they are equal to zero. Thus $\left(T_{i}-T_{s}\right)^{+}=0$ a.e. on $\mathrm{I}_{1}^{i}, i=1,2$, and by the Poincaré's inequality we deduce (6.15).

Remark 6.5. - A relationship between the parameters $h, k_{i}, T_{c}, T_{s}, n_{1}$ and $c$ ensuring that $S$ is indeed enclosed in $\Omega$ is an open problem. However, the following properties are easy to verify:

1) If $h n_{1}+\alpha\left(T_{s}-T_{c}\right)=0$ a.e. on $\Gamma_{1}^{i}, i=1,2$, then $T_{i}=T_{s}$, $q_{i}=1, i=1,2$ is a solution of (WP), and, consequently, the liquid phase fills up the whole domain.

2) If $h n_{1}+\alpha\left(T_{s}-T_{c}\right)<0$ a.e. on $\Gamma_{1}^{i}, i=1,2$, then there exists a subset of $\Omega$ where the temperature is greater than $T_{s}$. Therefore, the assumption (H6) is necessary in order to obtain solutions of the initial problem (P).

3) The following "onedimensional" problem gives us an insight into the shape of the solution of the problem (WP):

Let us consider the problem (WP) taking place in the domain $\Omega=[0,1] \times[0,1]$. Then $n_{1}=-1$ on $\Gamma_{1}^{i}, i=1,2$. We choose $h, \alpha$ and $T_{c}$ as three constants and $k_{i}(T)=1, i=1,2$. The solution is as follows:

i) For $h \leq \frac{\alpha\left(T_{s}-T_{c}\right)}{\alpha+1}$,

$$
\begin{aligned}
T\left(x_{1}, x_{2}\right) & =h x_{1}+\frac{\alpha T_{c}+h}{\alpha}, \\
q\left(x_{1}, x_{2}\right) & =0
\end{aligned}
$$


thus $T<T_{s}$ and no free boundary exists.

$$
\begin{aligned}
& \text { ii) For } \frac{\alpha\left(T_{s}-T_{c}\right)}{\alpha+1}<h \leq \alpha\left(T_{s}-T_{c}\right) \text {, } \\
& T\left(x_{1}, x_{2}\right)=\left\{\begin{array}{ccc}
h x_{1}+\frac{\alpha T_{c}+h}{\alpha} & \text { if } & x_{1} \leq \gamma \\
T_{s} & \text { if } & x_{1}>\gamma
\end{array}\right. \\
& q\left(x_{1}, x_{2}\right)=\left\{\begin{array}{lll}
0 & \text { if } & x_{1}<\gamma \\
1 & \text { if } & x_{1}>\gamma
\end{array}\right.
\end{aligned}
$$

where $\gamma$ is given by

$$
\gamma=\frac{\alpha\left(T_{s}-T_{c}\right)-h}{\alpha h}
$$

The free boundary is given by $S=\left\{\left(\gamma, x_{2}\right), 0 \leq x_{2} \leq 1\right\}$. iii) For $h>\alpha\left(T_{s}-T_{c}\right)$,

$$
\begin{aligned}
& T\left(x_{1}, x_{2}\right)=\frac{\alpha T_{c}+h}{\alpha}, \\
& q\left(x_{1}, x_{2}\right)=1
\end{aligned}
$$

and then condition $T \leq T_{s}$ does not hold. Thus the liquid phase fills up the whole domain.

\section{REFERENCES}

[1] Y. ARITA, N. URATA and H. IkEuChi, Estimation of Frozen Bath Shape in an Aluminium Reduction Cell by Computer Simulation, Light Metals, 1978, pp. 59-72.

[2] G. Bayada and M. Chambat, Existence and Uniqueness for a Lubrication Problem with Nonregular Conditions on the Free Boundary, Boll. U.M.I., Vol. 3-B 6, 1984, pp. 543-557.

[3] A. Bermúdez, L. Carpintero, M. C. Muñz and P. Quintela, An Inverse Problem Related to the Three-dimensional Modelling of Aluminium Electrolytic Cells. In L. C. Wrobel and C. A. Brebbia, editors, Computational Modelling of Free and Moving Boundary Problems II, Computational Mechanics Publications, 1993, pp. 377-388.

[4] A. Bermúdez, M. C. MuÑIz and P. Quintela, Numerical Solution of a Three-dimensional Thermoelectric Problem Taking Place in an Aluminium Electrolytic Cell, Computer Methods in Applied Mechanics and Engineering, Vol. 106, 1993, pp. 129-142.

[5] A. Bermúdez, M. C. MuÑzz and P. Quintela, Numerical Solution of a Stefan Problem Arising in the Thermoelectrical Modelling of Aluminium Electrolytic Cells. In L. C. Wrobel and C. A. Brebbia, editors, Computational Modelling of Free and Moving Boundary Problems, Vol. 2, Computational Mechanics Publications/De Gruyter, 1991, pp. 39-58.

[6] A. Bermúdez, M. C. Muñiz and P. Quintela, Existence and Uniqueness for a Free Boundary Problem in Aluminium Electrolysis, Journal of Mathematical Analysis and Applications, Vol. 191, 1995, pp. 497-527.

[7] H. BRezls, Analyse Fonctionnelle. Théorie et Applications, Masson, Paris, 1983. 
[8] H. Brezis, D. Kinderlehrer and G. Stampacchia, Sur une Nouvelle Formulation du Problème de L'écoulement à Travers une Digue, C. R. Acad. Sc. Paris, Vol. A 287, 1978, pp. 711-714.

[9] J. Carrillo-Menéndez and M. Chipot, On the Dam Problem, Journal of Differential Equations, Vol. 45, 1982, pp. 234-271.

[10] J. Dukany and C. VázQuez, Numerical Approach of Lubrication Problems in Journal Bearing Devices with Axial Supply. In Ch. Hirsch et al., editors, Numerical Methods in Engineering '92, Elsevier Science Publishers B.V., 1992, pp. 839-844.

[11] K. Grjotheim, C. Krohn, M. Malinovsky, K. Matiašovsky and J. Thonstad, Aluminium Electrolysis, Aluminium Verlag, Dusseldorf, 1982.

[12] K. Grjotheim and H. Kvande, Understanding the Hall-Héroult Process for Production of Aluminium, Aluminium Verlag, Dusseldorf, 1986.

[13] S. D. Howison, J. F. Rodrigues and M. SHLlor, Stationary Solutions to the Thermistor Problem, J. Math. Anal. Appl., Vol. 174, 1993, pp. 573-588.

[14] D. Kinderlehrer and G. STAMPACCHIA, An Introduction to Variational Inequalities and Their Applications, Academic Press, 1980.

[15] M. C. MuÑiz, Estudio Matemático de un Problema de Stefan Relacionado con la Modelización Termoeléctrica de Cubas de Electrolisis de Aluminio, PhD thesis, Universidad de Santiago de Compostela, 1995.

[16] T. RoubícEk, The Stefan Problem in Heterogeneous Media, Ann. Inst. Henri Poincaré, Vol. 6(6), 1989, pp. 481-501.

[17] C. Saguez, Un Problème de Stefan avec Source sur la Frontière Libre, Rapport de Recherche INRIA n. 268, Rocquencourt, 1977.

[18] M. P. TAYLOR and B. J. WelCh, Melt/freeze Heat Transfer Measurements in Cryolite-based Electrolytes, Metallurgical Trans., Vol. B. 18, 1987, pp. 391-398.

(Manuscript received January 30, 1996;

Revised August 26, 1996.) 